\title{
Switchable Stimuli-Responsive Heterogeneous Catalysis
}

\author{
Irene Vassalini ${ }^{1,2, *}$ and Ivano Alessandri $1,3,4, *$ (D) \\ 1 Consorzio Interuniversitario Nazionale per la Scienza e la Tecnologia dei Materiali (INSTM)—Research \\ University of Brescia, via Branze 38, 25123 Brescia, Italy \\ 2 Chemistry for Technologies Laboratory, Mechanical and Industrial Engineering Department, \\ University of Brescia, via Branze 38, 25123 Brescia, Italy \\ 3 National Institute of Optics-Italian National Research Council (INO-CNR), via Branze 38, 25123 Brescia, Italy \\ 4 Department of Information Engineering, University of Brescia, via Branze 38, 25123 Brescia, Italy \\ * Correspondence: i.vassalini001@unibs.it (I.V.); ivano.alessandri@unibs.it (I.A.); Tel.: +39-030-3715475 (I.A.)
}

Received: 29 October 2018; Accepted: 16 November 2018; Published: 22 November 2018

\begin{abstract}
Heterogeneous catalytic systems based on the use of stimuli-responsive materials can be switched from an "on" active state to an "off" inactive state, which contributes to endowing the catalysts with unique functional properties, such as adaptability, recyclability and precise spatial and temporal control on different types of chemical reactions. All these properties constitute a step toward the development of nature-inspired catalytic systems. Even if this is a niche area in the field of catalysis, it is possible to find in literature intriguing examples of dynamic catalysts, whose systematic analysis and review are still lacking. The aim of this work is to examine the recent developments of stimuli-responsive heterogeneous catalytic systems from the viewpoint of different approaches that have been proposed to obtain a dynamic control of catalytic efficiency. Because of the variety of reactions and conditions, it is difficult to make a quantitative comparison between the efficiencies of the considered systems, but the analysis of the different strategies can inspire the preparation of new smart catalytic systems.
\end{abstract}

Keywords: switchable catalysis; heterogeneous catalysis; smart materials; stimuli-responsive; on-demand catalysis

\section{Introduction}

Finding catalysts with high catalytic activity, selectivity, recyclability and that are, at the same time, non-toxic, cheap and easily available, is a crucial step to meet the current energetic requests and constraints. Some of these features (high activity and selectivity) are typical of natural catalytic systems, e.g., enzymes, which are also able to modulate their activity dynamically and perform catalysis in response to the application of an external stimulus. For example, they can change, on demand, their internal structure and their functionalities in response to the presence of substrates, inhibitors, cofactors and/or as a consequence of variations of $\mathrm{pH}$ or ionic strength. Including this dynamic behavior in synthetic catalytic systems would be a further progress toward efficient smart catalysis. Thanks to the intrinsic will of scientists to understand and mimic nature, different types of synthetic stimuli-responsive systems have been developed over the time, creating a large palette of "smart" materials [1,2]. They can modify one or more properties in a controlled fashion using externally delivered energy and they have the ability to restore their original state once the stimulus has been removed, behaving in a truly dynamic way. According to the stimulus to which they are able to respond to, different systems have been prepared: $\mathrm{pH}$-responsive [3], temperature-responsive and photo-switchable materials [4] are some examples, but other stimuli, such as electric fields [5], magnetic 
fields [6] and mechanical stress [7], have also been exploited. A large variability can also be observed in the type of system response: variations of chemical properties, solubility, shape, volume, color, aggregation state, electrical properties, magnetic properties, mechanical properties, etc. are all possible. Thanks to this high versatility, artificial smart materials have found application in various sectors [8], ranging from medicine to national security and structural engineering [9]. They are commonly employed for drug delivery [10], self-healing [11], sensing [12], actuations [13], information storage [14], etc. Their use for catalytic purposes has been remarkably less investigated, even if heterogeneous catalysis can fully take advantage of the dynamic behavior of smart materials. For example, it is possible to make a catalyst to pass from a state of activity (an "on" state) to a state of inertness (an "off" state) in a controlled way, with a precise temporal control, obtaining switchable catalysis. In other circumstances, the possibility of modulating the reaction rate in a continuous way, regulating the amount of accessible active sites and accelerating or lowering the reaction rate in a non-discrete way, is more interesting, and in this case we can also exploit stimuli-responsive materials. It is also possible to create microenvironments in which the reaction occurs preferentially, accomplishing a spatial control on the reaction development. In addition, the responsiveness of the employed systems can be exploited to improve the recyclability of the catalytic system, making the catalyst separation from the reaction medium and its reuse easier.

In this review, we analyze some of the latest works on dynamic systems in the field of heterogeneous catalysis. Many more examples can be found for homogeneous catalysis, but most of them have been already analyzed in previous works [15-18]. Because of the variety of reactions and conditions considered in the different reported systems, the final aim of this review is to analyze the different approaches followed to obtain a dynamic control on their catalytic properties, rather than comparing their efficiency from a quantitative point of view or analyzing the chemical mechanism at the basis of the different catalyzed reactions. In particular, independently of the employed stimulus, reaction to be catalyzed and type of catalyst, it is possible to identify two main strategies that are followed to pursue dynamic/switchable catalysis: (a) the applied stimulus modifies the accessibility of the active sites of the catalytic system, by either varying the diffusion dynamic of chemical species (reactants, inhibitors or activators) towards the catalyst (Figure 1a,b) or changing the aggregation state of the catalyst (Figure 1c,d); (b) the applied stimulus produces a variation of the nature of active sites of the catalyst (Figure 1e). In most cases, high catalytic activity is obtained by promoting the free diffusion of reactants towards the catalyst surface; on the contrary, in order to slow down or stop the reaction, their diffusion has to be hindered. The aggregation state of the catalyst can also be utilized to control the reaction rate. For example, in most reported cases, a high catalytic activity can be obtained when the catalyst is uniformly distributed inside the reaction medium in a non-aggregate state, while the reaction rate can be reduced and the reaction occurrence blocked when the catalyst aggregates. Anyway, examples of reaction rate enhancement as a consequence of catalyst aggregation can be found.

Because of the importance of the features that can be obtained in dynamic catalytic systems, many efforts have been continuously made in order to extend them also to conventional systems and pursue smart catalysis characterized by recyclability, long-term stability and self-regeneration. New strategies have recently been proposed, in particular, thanks to the insertion inside the catalytic system of switchable active sites, which are able to respond to an external stimulus and restore their catalytic activity after deactivation (Figure 1e). In this case, the external stimulus is able to change the nature of active sites present on the catalyst surface and regenerate them.

In the following, we will review the progress in stimuli-responsive heterogeneous catalytic systems from the perspective of the different proposed strategies (control of diffusion, modification of aggregation state and the regeneration of active sites). An additional section focused on the description of macroscopic dynamic devices used for catalytic purposes will be presented. Finally, the conclusions will underline the advantages and limitations of current smart catalytic systems and future challenges. 


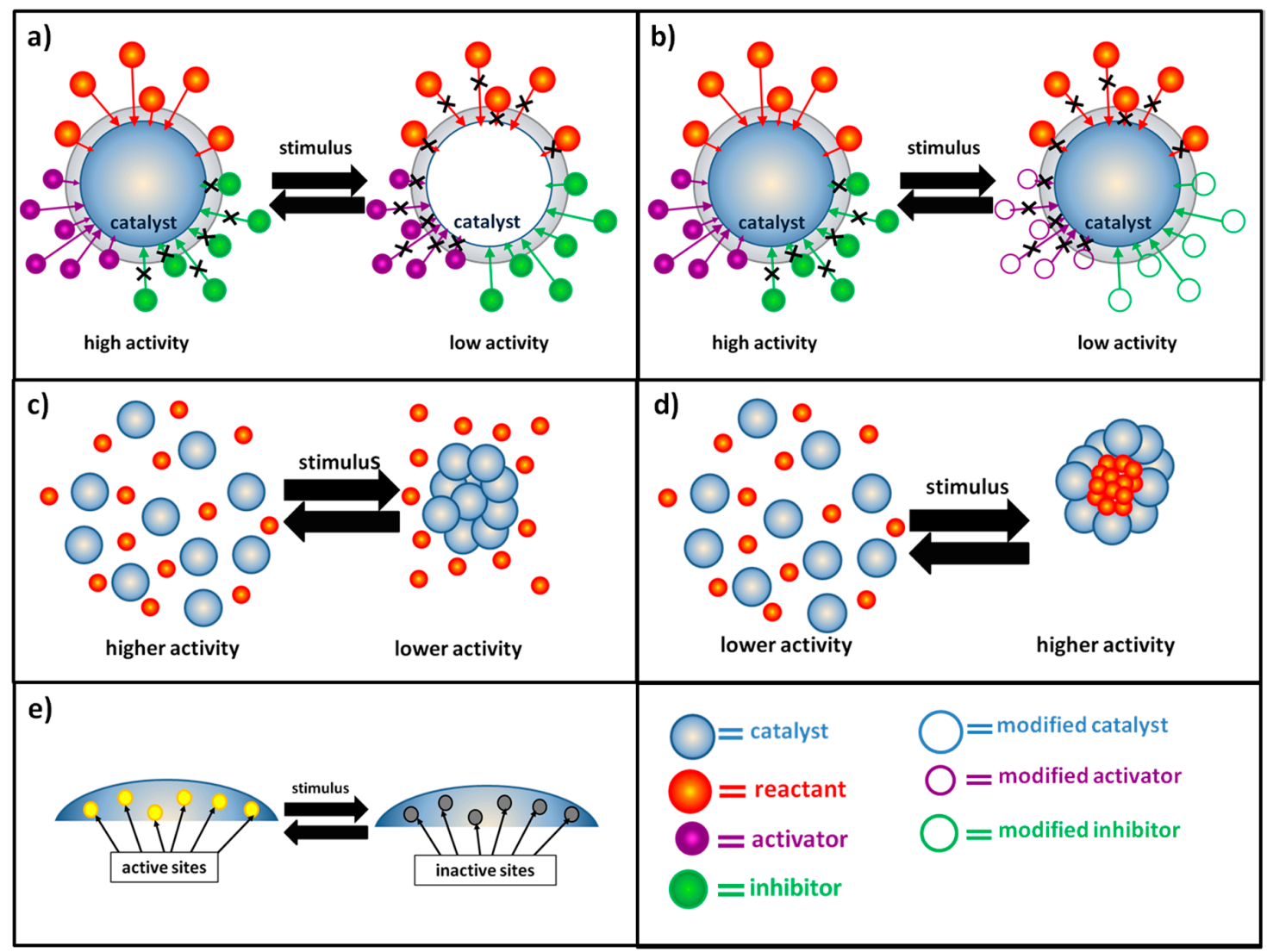

Figure 1. Schematic representation of the different strategies proposed to obtain switchable catalysis. (a) Controlling the reaction rate and the catalytic activity of the system by means of the variation of diffusion dynamics of reactants, activators or inhibitors toward the catalyst surface because of stimuli-induced modification of the catalyst itself; (b) Controlling the reaction rate and the catalytic activity of the system by means of the variation of diffusion dynamics of the reactants, activators or inhibitors toward the catalyst surface because of stimuli-induced modification of the chemical species in solution (activators or inhibitors); (c) Controlling the reaction rate and the catalytic activity of a catalyst by means of modification of its aggregation state. Higher catalysis is achieved when the catalyst is homogeneously distributed inside the reaction medium, while it is lowered after its aggregation. This is a surface effect; (d) Controlling the reaction rate and the catalytic activity of a catalyst by means of modification of its aggregation state. Higher catalysis is achieved when the catalyst is in its aggregated state, because it is able to trap reactants in preferential microenvironments. Lower catalytic efficiency is achieved after the disruption of these aggregates; (e) Controlling the reaction rate and the catalytic activity of a catalyst by means of modification of the nature of active sites on the catalyst surface.

\section{Switchable Catalysis by Means of Control of Diffusion Dynamics and Aggregation State of the Catalyst}

The most widespread strategy to obtain switchable catalysis is the preparation of systems able to respond to the application of an external stimulus by modifying the diffusion dynamics of different chemical species towards the catalyst surface. An enhancement of the catalytic activity and reaction rate is obtained when, after the stimulus, the diffusion of reactants or activators (i.e., the chemical species that promote the interaction between the catalyst and its substrates) is facilitated and the diffusion of inhibitors is obstructed. On the contrary, the reaction rate can be lowered and, eventually, the reaction can be stopped when the diffusion of reactants and activators is hindered and the diffusion of inhibitors is favored (Figure 1a,b). Variation of the diffusion dynamics can be obtained both in cases where the external stimulus causes a direct modification of the catalytic system (generally metallic nanoparticles embedded in a stimuli-responsive hydrogel matrix or metallic nanoparticles functionalized with 
stimuli-responsive units) (Figure 1a) or when the external stimulus modifies the properties of chemical species in the reaction medium (Figure 1b). The first approach is the most utilized, and in the majority of cases, the external stimulus is used to modify the properties (hydrophilicity, polarity, solubility and volume) of the polymeric matrix with which the catalyst is combined. In both cases, different examples can be found using a variety of stimuli, especially temperature, $\mathrm{pH}$ or light. By using the same stimuli, it is possible to control the catalytic activity of the system also by means of variations of the aggregation state of the catalytic system. In most of the reported cases in the literature, a high catalytic activity is observed when the catalyst is homogeneously distributed inside the reaction medium in a non-aggregate state and a decrease of the reaction rate is obtained after its aggregation. This results from a surface effect: in the non-aggregate state catalyst surface is higher, and there are many more active sites accessible to the reactants (Figure 1c). However, some interesting examples of reaction rate enhancement as a consequence of catalyst aggregation can be found. In particular, this is due to a confinement effect: thanks to the catalyst aggregation, preferential microenvironments for the occurrence of the reaction are formed. As a consequence of the catalyst aggregation, the reactants are concentrated and "trapped" in small regions, characterized by high catalytic activity (Figure 1d). In most cases, it is difficult to distinguish whether the external stimulus modifies the diffusion dynamic of chemical species or the aggregation state of the catalytic system, often the two effects are combined and the overall result is a switchable catalysis based on switchable accessibility of catalytic active sites.

\subsection{Temperature-Controlled Switchable Catalysis}

Temperature is the stimulus that has been most widely investigated, and different dynamic catalytic systems based on the employment of temperature-responsive polymers have been proposed. In most cases, a dynamic behavior is obtained by exploiting the capability of Poly( $N$-IsoPropyl AcrilAmide) PNIPAm to undergo a reversible phase transition at $32{ }^{\circ} \mathrm{C}$ [19]. This temperature is called Lower Critical Solution Temperature (LCST). Below and above LCST, many polymer properties change: solubility in water, transparency, volume and morphology. In particular, at T < LCST, PNIPAm is hydrophilic, soluble in water and it is in a swollen state. It is in the form of coils and its solutions are transparent. On the contrary, at T > LCST, PNIPAm is hydrophobic, insoluble in water, and it is in a shrunken state. In this condition it forms globules, tends to precipitate and it generates turbid solutions. These properties can be exploited also in the field of catalysis: the catalyst activity can be switched "on" and "off" by adjusting the temperature below and above the LCST, changing the hydrophilicity of the polymeric matrix with which the catalyst is combined and converting it from an hydrophilic diffusion layer (easily accessible to polar molecules) to an hydrophobic diffusion layer (difficultly accessible to polar molecules). In addition, this behavior can be exploited to facilitate the recovery of the catalytic system, modifying the aggregation state of the polymeric matrix from a homogeneous state (when $\mathrm{T}<\mathrm{LCST}$ ) to a heterogeneous one (when T $>$ LCST).

For example, Ballauff and coworkers [20] reported the use of silver nanoparticles embedded in a polymeric network of PNIPAm cross-linked with $N, N^{\prime}$-methylenebisacrylamide (BIS) attached to a colloidal core made of polystyrene (PS) to catalyze the reaction of reduction of 4-nitrophenol by $\mathrm{NaBH}_{4}$ to 4 -aminophenol in water. When the PS-PNIPAm-Ag system is at T $<$ LCST, the PNIPAm chains are hydrophilic, the shell network is swollen, and the metallic nanoparticles are fully accessible to reactants. In these conditions, the reduction of 4-nitrophenol is well catalyzed. When temperature is enhanced to T > LCST, a significant decrease of the reaction rate is recorded. In these conditions, PNIPAm becomes hydrophobic, the polymeric network shrinks and the diffusion of the reactant within the network is hindered. As visible in Figure 2, by increasing the temperature, the hydrodynamic radius of PS-PNIPAm-Ag particles $\left(\mathrm{R}_{\mathrm{H}}\right)$ lowers, indicating that the PNIPAm layer gradually shrinks and this behavior affects the catalytic efficiency of the system. In fact, instead of a simple linear relationship between $\ln k$ and $\mathrm{T}^{-1}$, the change of rate constant $(k)$ with temperature can be divided in three regions. At low temperature (green square region in Figure 2), $k$ exhibits a conventional Arrhenius-type dependence on T, since the PNIPAm network is totally swollen with water and AgNPs 
are fully accessible to reactants. When $25^{\circ} \mathrm{C}<\mathrm{T}<32^{\circ} \mathrm{C}$ (yellow square region in Figure 2), the PNIPAm network begins to shrink and a diffusional barrier for reactants is formed around AgNPs, which turns down the reaction rate and this effect over-compensates for the thermodynamic increase of $k$ by the rise in temperature. This behavior is maintained until the LCST is reached, when $k$ acquires its minimum value. At T > LCST (red square region in Figure 2), instead, the density of the polymer network is constant and the strong increase in $k$ with $\mathrm{T}$ dominates and the reaction rate rises again.

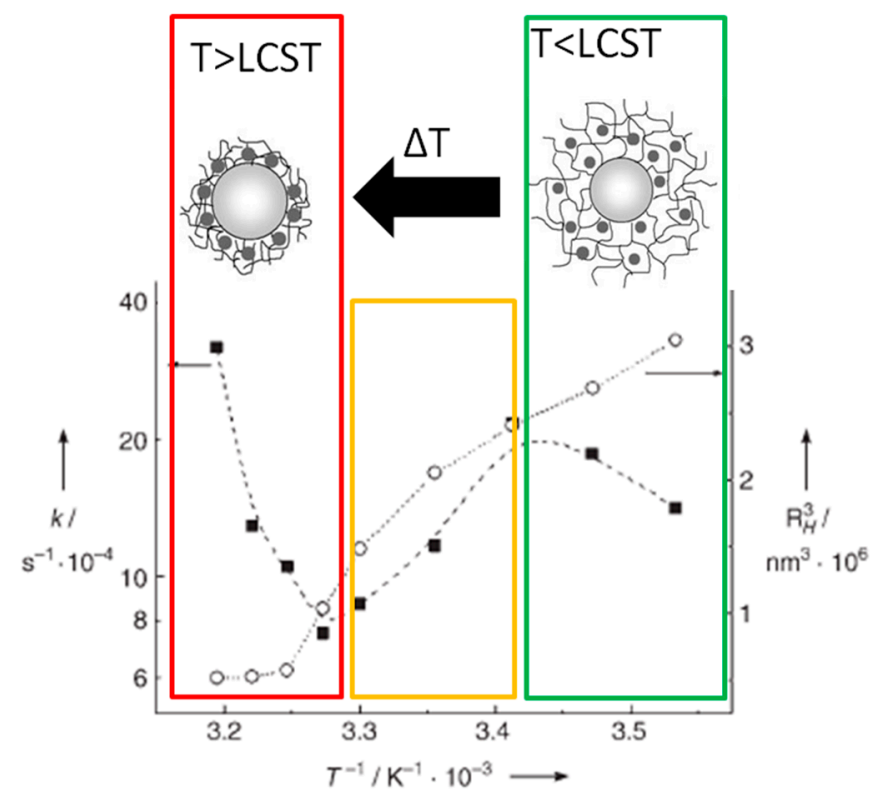

Figure 2. Arrhenius plot of the reaction rate and catalyst volume: black squared dots represent reaction rate constant $k$ measured in the presence of the PS-PNIPAm-Ag systems at different temperatures and white dots indicate the volume change of the catalytic particles with temperature. Adapted and reprinted with permission from ref. [20]. Copyright (2006) Wiley.

Similar behaviors have been obtained by combining PNIPAm with other copolymers and other types of metallic nanoparticles. For example, Shi and coworkers showed that it is possible to prepare switchable catalysts by creating hydrogels of poly(glycidyl methacrylate) (PGMA) and PNIPAm (PGMA-co-PNIPAm) and incorporating AuNPs [21], stabilizing gold nanoparticles with PNIPAm- $b$ P4VP (poly(4-vinyl pyridine) [22] or supporting gold nanoparticles on PAA/P4PV/PEG/PNIPAm [23] micelles. PNIPAm hydrogels can be combined also with catalysts different from metallic nanoparticles. For example, Huang et al. [24] reported that it is possible to introduce glutathione peroxidase (GPx) active sites (functional monomer bis-(3-acryloyloxypropyl)-telluride) into a PNIPAm scaffold and obtain a smart microgel catalyst for the reduction of cumene hydroperoxide (CUOOH) by 3-carboxyl-4-nitrobenzenethiol. In this way, it is possible to create a dynamic artificial catalyst mimicking the activity of an antioxidative enzyme that plays a crucial role in the control of reactive oxygen species (ROS) in biological systems. In this regard, it is important to underline that PNIPAm undergoes a temperature-responsive behavior at a near-physiological temperature.

Actually, a similar behavior can also be obtained by employing other temperature-responsive polymers different from PNIPAm. For example, Liu et al. [25] reported the use of AuNPs functionalized with citrate and a thermo-responsive hyperbranched polyethylenimine with isobutyramide (HPEI-IBAm) for the catalysis of the reduction of 4-nitrophenol by $\mathrm{NaBH}_{4}$. The most interesting aspect of their work lies in the fact that with their system it is possible to modulate the LCSTs of the thermo-responsive AuNPs over a broader range $\left(20-50^{\circ} \mathrm{C}\right)$ of temperature. The same is true for the activation/deactivation of the catalyst. As in the above-cited examples, the activity of the catalyst is reduced by enhancing the temperature above the LCST of system, but different strategies have 
been identified to regulate the value of LCST: alteration of the molecular weight of the HPEI core, modification of the degree of substitution of the IBAm groups of the HPEI-IBAm polymers, or variation of solution $\mathrm{pH}$.

All these systems show an inverse temperature response: their reactivity decreases when temperature increases. Cao and coworkers [26], instead, proposed nanoreactors made of Ag nanoparticles and a functional polymer composite of poly(acrylamide) (PAAm) and poly(2-acrylamide2-methylpropanesulfonic acid) (PAMPS) with positive responsive function. This means that its catalytic activity significantly increases with temperature enhancement. In particular, in addition to the thermodynamic enhancement of the reaction rate with temperature increase, an abrupt change is recorded in correspondence of a transition temperature (Figure 3a). This effect is clearly evident when the catalytic activity of the Ag-PAAm-PAMPS system is compared with that of an analogous system made of Ag-PAAm. The catalytic activity of both the systems increases with temperature enhancement, but for Ag-PAAm-PAMPS a great increase of the $\%$ of reactant conversion occurs at about $25-35{ }^{\circ} \mathrm{C}$. In fact, at $20^{\circ} \mathrm{C}$, Ag-PAAm-PAMPS demonstrates relatively low catalytic activity that is much smaller than that of Ag-PAAm; on the contrary, at $40^{\circ} \mathrm{C}$ the reactivity of Ag-PAAm-PAMPS increases to a much higher level, which is close to that of Ag-PAAm. Interestingly, the temperature range in which this modification occurs $\left(25-35^{\circ} \mathrm{C}\right)$ is in agreement with the temperature range in which a dramatic enhancement of the hydrodynamic radius of Ag-PAAm-PAMPS is recorded (Figure $3 b$ ). The authors suggested that thermal sensitivity derives from the PAAm-PAMPS interactions: at low temperature there are strong electrostatic interactions between the $\mathrm{NH}_{3}{ }^{+}$groups of $\mathrm{PAAm}$ and $\mathrm{SO}_{3}{ }^{-}$ groups of PAMPS; at high temperature, instead, a dissociation of the interpolymer complexation occurs (Figure 3c).

a)

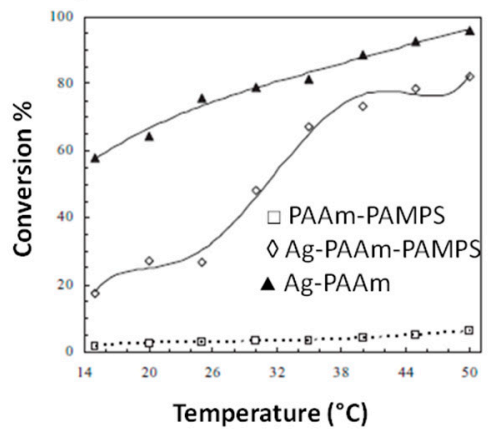

b)

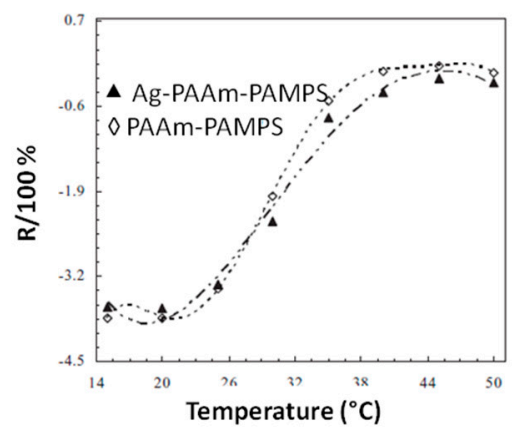

c)

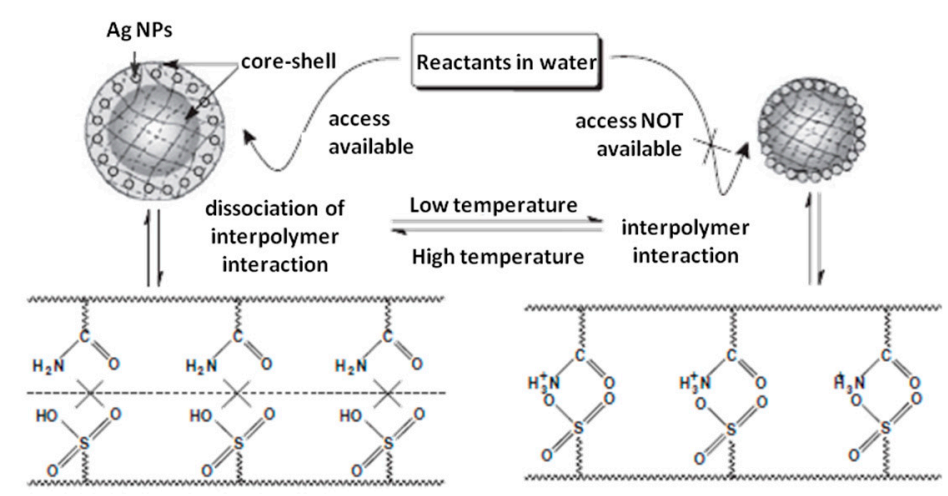

Figure 3. (a) \% of conversion of 4-nitrophenol to 4-aminophenol after $30 \mathrm{~min}$ of reaction at different temperatures, using different systems as catalyst, (b) Hydrodynamic radius of the catalytic system as a function of temperature; (c) Proposed mechanism for Ag NPs-PAAm-PAMPS nanoreactors with a temperature positive responsive function. Reprinted with permission from ref. [26]. Copyright (2010) Wiley. 
As a consequence, at low temperatures (e.g., $20^{\circ} \mathrm{C}$ ), the PAA-PAMPS polymeric shell is compact and restricts the access of reactants to the encapsulated Ag NPs, leading to a low catalytic activity of the system. On the contrary, at relatively high temperatures (e.g., $40^{\circ} \mathrm{C}$ ), a significant catalytic activity can be achieved thanks to the dissociation of the interpolymer complexation, which causes relaxation and swelling of the shell network and allows reactants to access the encapsulated AgNPs. By lowering temperature below the transition temperature, it is possible to decrease again the reaction rate. Very recently, Borrmann et al. [27] proposed another microgel catalyst characterized by a positive temperature response which has been successfully tested for the desymmetrization of meso-anhydrides in organic solvents.

\subsection{Switchable Catalysis Based on Variation of $p H$, Ionic Strength or Other Chemical Stimuli}

Similarly, pH-responsive polymers can be employed to functionalize metallic nanoparticles and obtain systems whose catalytic activity can be switched on and off by adjusting $\mathrm{pH}$ or ionic strength of the reaction medium. Generally speaking, $\mathrm{pH}$-responsive polymers are polyelectrolytes with weakly ionizable groups in their structure, which either accept or release protons in response to modification of environmental $\mathrm{pH}$. Both weak polyacids and polybases exist. Polyacids accept protons at low values of $\mathrm{pH}$ and release them at neutral or high values of $\mathrm{pH}$, acquiring negative charges that create electrostatic repulsion and cause swelling of the polymer network. Examples are poly(acrylic acid) (PAA) or poly(methacrylic acid) (PMMA). In the case of polybases, instead, they are protonated and uncharged at high values of $\mathrm{pH}$ and they are positively ionized in neutral and acidic conditions. In this case, the positive charges inside the polymeric network at $\mathrm{pH}$ values lower than the polymer $\mathrm{pk}_{\mathrm{a}}$ cause electrostatic repulsion and polymer swelling. Examples are poly(4 or 2-vinylpyridine) (P4VP or P2VP) and poly(1-vinylimidazole)(PVIm). Also, in this case, the most frequently employed strategy for the control of the dynamic behavior of the system catalytic activity is based on the control of the swelling degree of the polymeric network used to functionalize the catalyst itself. When the polymeric network is in a swollen state, the reactants are free to reach the catalyst surface; on the contrary, when the polymeric network is in a shrunken state, reactant diffusion is hindered, and the reaction is less catalyzed. An example is the work of Xiao et al. [28], who reported the preparation of Au@PVP hybrid nanogels for the $\mathrm{pH}$-responsive catalysis of the reduction of 4-nitrophenol by $\mathrm{NaBH}_{4}$. Actually, this reaction is normally affected by $\mathrm{pH}$ variations; in particular, during the process of reduction, sodium metaborate is produced, causing an increase in $\mathrm{pH}$, and when it reaches high values, such as 9-10, 4-nitrophenolate ions can be produced [29]. Therefore, it is necessary to consider that a lowering of $\mathrm{pH}$ by itself favors the reaction of reduction and that 4-nitrophenolate can react differently from 4-nitrophenol. All these aspects make the study of reaction kinetics variation difficult, but the authors showed that the system is characterized by a pH-responsive swelling-deswelling behavior (variations of hydrodynamic radius), which is able to influence also the catalytic activity of the system.

When $\mathrm{pH}$-responsive polymers are involved, it is possible to achieve a dynamic control on their behavior also by varying the ionic strength of the reaction medium. An example was proposed by Yang and co-workers [30]. In this case, the reduction of 4-nitrophenol by $\mathrm{NaBH}_{4}$ is catalyzed by Ag@air@PMAA nanorattles. The system is composed of a core made of AgNPs, surrounded by an empty layer of air, which serves as microreactor, and a stimuli-responsive PMMA layer (Figure 4a). At $\mathrm{pH}$ 9, all the PMMA chains are deprotonated and negatively charged: they repel each other and the polymer shell is in a swollen state, which promotes the diffusion of reactants inside the air cavity, where the reaction is efficiently catalyzed. By adding $\mathrm{NaCl}$ inside the reaction medium, $\mathrm{Na}^{+}$ions are released and interact with the $\mathrm{COO}^{-}$groups of the PMAA chains. In this way, the above-cited electrostatic repulsion is reduced, the polymeric structure collapses and the diffusion of reactants towards the Ag core is hindered. The result is a decreased catalytic activity in the system, similar to the one that can be obtained by changing the solution $\mathrm{pH}$. In addition, by modifying the ionic strength of the solution it is possible not only to switch the catalysis between an "on" and "off" state, but also to control, dynamically, the reaction rate in a continuous way (Figure $4 b$ ). 

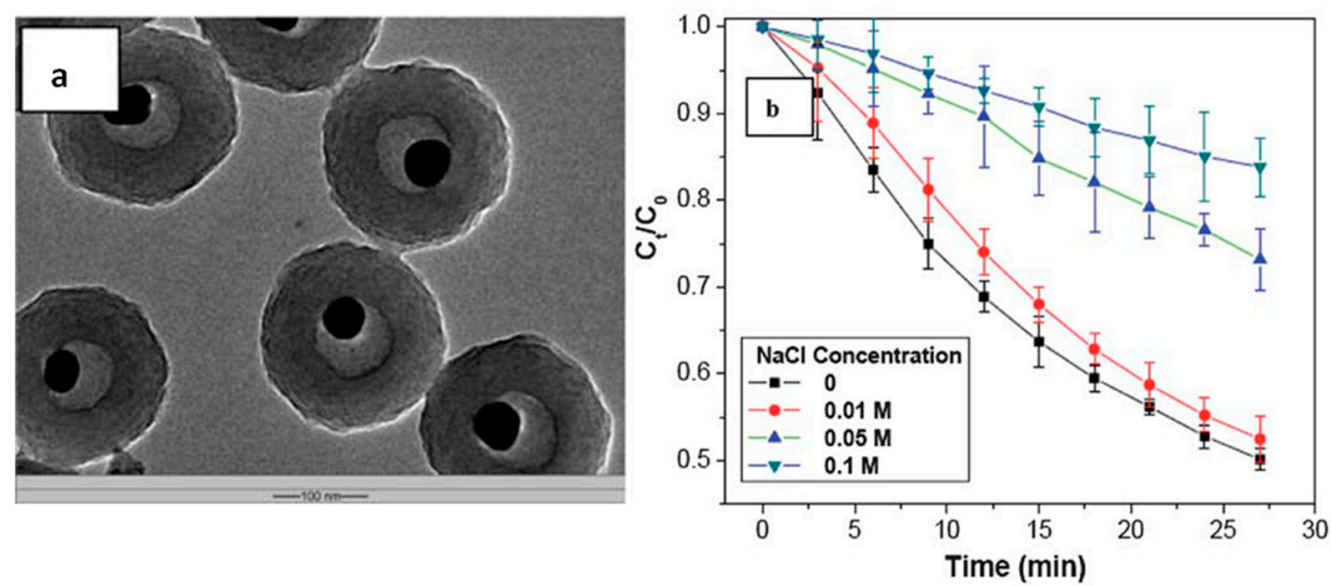

Figure 4. (a) TEM micrograph of the Ag@air@PMAA hybrid nanorattles; (b) variation of the reaction kinetics of $p$-nitrophenol reduction by the Ag@air@PMAA nanorattles under the effect of different salt concentrations in the reaction medium $\left(\mathrm{pH} 9.2, \mathrm{C}_{0}\right.$ and $\mathrm{C}_{\mathrm{t}}$ are the initial and instantaneous concentration of $p$-nitrophenol, respectively). Adapted and reprinted with permission from ref. [30]. Copyright (2011) Royal Society of Chemistry.

Zhang and coworkers suggested that the capability of the polymeric matrix to pass from an hydrophilic to an hydrophobic state according to the external $\mathrm{pH}$ can also be exploited to facilitate the separation and reuse of the whole catalytic system [31,32], similarly to what happens when $\mathrm{pH}$-sensitive organic capping agents are used to functionalize metallic nanoparticles [33]. In fact, besides the modification of the capability of permeation of reactants through the matrix toward the catalyst surface, the variable degree of hydrophilicity of the polymeric network of the composite systems enables to modify the aggregation state of the polymeric matrix in which the catalysts are embedded. In particular, they showed that it is possible to obtain efficient recyclable catalytic systems for the Suzuki and Heck reactions in the case of iminodiacetic (IDA)-functionalized Pd nanoparticles embedded in the shell layer of core-shell PS-PMMA [31] or PS-PGMA [32] systems. In fact it is possible to disperse PS-co-PMAA-IDA-Pd in basic reaction medium $(\mathrm{pH}>10)$ and cause it to precipitate by lowering the $\mathrm{pH}$ below 5 . In basic environments, the system behaves as a homogeneous catalyst with reactants that freely reach the catalyst surface, so it is characterized by a very high catalytic activity. By lowering the $\mathrm{pH}$, the polymeric matrix becomes more hydrophobic, the diffusion of reactant is hindered, and the catalytic activity of the whole system is reduced. In addition, in acidic environments, the polymers tend to segregate from water, the system precipitates and becomes a heterogeneous catalyst, which can be easily removed from the solution. Subsequently, it can be resuspended in a fresh reaction medium and efficiently reused once $\mathrm{pH}$ has been adjusted at values higher than 10 . They showed that the same system can be reused 4 times without any loss of activity. A similar behavior is ascribed also to the PS-co-PGMA-IDA-Pd system, even if in this case the $\mathrm{pH}$ values for the phase transition are 3 and 11. Also Pd NPs supported on PS-co-PAEMA-co-PMMA core/shell NPs [34] behave in a similar way.

$\mathrm{pH}$ (as well as temperature) variations can be used also to enhance the reaction rate by inducing reactant confinement within polymeric matrix enriched of catalytic NPs. For example, Wang et al. [35] reported that it is possible to catalyze C-C Cross-Coupling reactions with Pd NPs embedded into PNIPAm-co-PMACHE hydrogels. By increasing the temperature of the system above the LCST of PNIPAm or by adjusting the $\mathrm{pH}$ above 10 or below 4 (PMACE swells at the $\mathrm{pH} 4-10$ and deswells out of this range), it is possible to make the hydrogel shrink, creating a hydrophobic environment ideal for hosting hydrophobic molecules. In addition, since Pd NPs are embedded into the hydrogel, when it shrinks, Pd catalysts are enriched and concentrated in a small volume (Figure 5). This fact, together with encapsulation of hydrophobic reactants and their concentration in a smaller volume rich of catalytic sites, makes it possible to obtain higher reaction rates. 


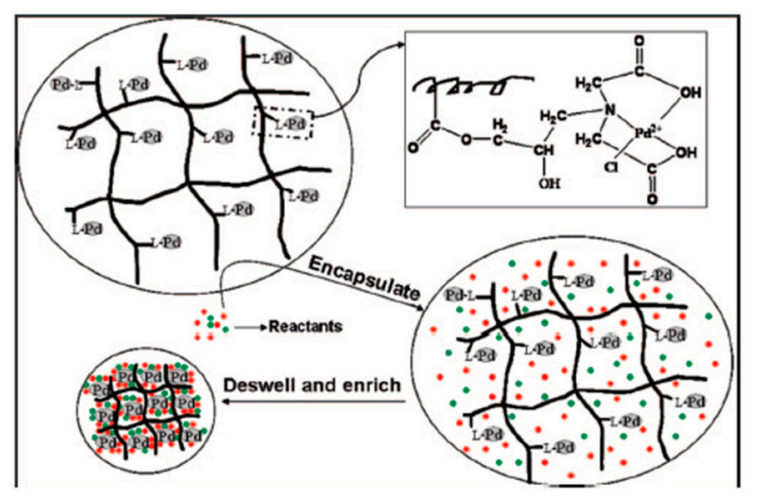

Figure 5. Schematic structure of the PNIPAm-co-PMACHE hydrogel/Pd(II) composite and representation of the processes of encapsulation and enrichment of the Pd catalyst and reactants within the hydrogel. Reprinted with permission from ref. [35]. Copyright (2009) American Chemical Society.

The enhancement of the reaction rate during hydrogel deswelling has been proved for the Suzuki and the Heck reactions, but a similar behavior was obtained by Zhang and coworkers [36] during the preparation of a composite catalyst for aerobic alcohol oxidation made of Au NPs embedded into PNIPAm-co-PMACHE hydrogel. In both the cases, the reversible swelling/deswelling of the hydrogel can be exploited also to obtain an easy separation and reuse of the catalytic system.

Variations of $\mathrm{pH}$ can be used also to create preferential channels to facilitate the diffusion of reactants towards the catalyst surface. The Shi group proposed an example [37]: core-shell-corona Au-micelle composites containing a PS core, a hybrid shell of P4P/Au/PEG and a PEG (polyethylene glycol) corona. At low values of $\mathrm{pH}($ (e.g., $\mathrm{pH}=2$ ), PEG and P4VP are hydrophilic and the hybrid shell is in a swollen state. This means that Au NPs are accessible to reactants and a good catalytic activity can be reached. By increasing $\mathrm{pH}$, the catalytic activity can be further enhanced. In fact, at $\mathrm{pH} \geq 7$, P4P become hydrophobic and collapses to the PS core, while PEG maintains its hydrophilic behavior. The hydrophilic PEG chains connect the PS core and pass through the collapsed hybrid shell, creating preferential channels for the diffusion of small hydrophilic reactants.

Dynamic catalysis can be obtained by switching the hydrophilicity/hydrophobicity of the systems by means of other chemical stimuli. For example, Byun et al. [38] have proposed a $\mathrm{CO}_{2}$-switchable catalyst for the photodegradation of organic dyes and photoredox reactions of organic water-soluble molecules under visible light illumination. The photocatalyst is a tertiary amine tethered conjugated polymer (P-BT-DEA), which exhibits a reversible hydrophilicity triggered by $\mathrm{CO}_{2}$. In fact, diethylamine (DEA) at the terminal of the polymer is able to react with $\mathrm{CO}_{2}$ dissolved in water producing the bicarbonate salt, which boosts the water compatibility of the polymeric chain, leading to good polymer dispersion in water. The adsorbed $\mathrm{CO}_{2}$ can be removed through $\mathrm{N}_{2}$ bubbling and the elimination of the bicarbonate salt causes precipitation of the hydrophobic polymer. As a consequence, in the presence of $\mathrm{CO}_{2}$ it is possible to use the system to efficiently catalyze the photodegradation of different organic dyes (rhodamine $B$, methylene blue and crystal violet) in water and various organic transformation reactions (e.g., photooxydation of 2-furoic acid, photoreduction of 4-nitrophenol and coupling of caffeine and aryldiazoniumtetrafluoroborate); on the contrary, when $\mathrm{N}_{2}$ is purged, it displaces $\mathrm{CO}_{2}$, the polymer becomes hydrophobic, it precipitates and its catalytic activity is reduced.

Another example of $\mathrm{CO}_{2}$-switchable polymer was reported by Zhang et al. [39] for the 4-nitrophenol reduction by $\mathrm{NaBH}_{4}$ in the presence of AuNPs functionalized with thiol-terminated PDEAEMA (poly( $N, N$-diethylaminoethylmethacrylate)). Without $\mathrm{CO}_{2}$, the PDEAEMA polymer is poorly soluble in water and polymeric chains are hydrophobic, they tend to contract, entangle and strongly interact with each other. As a consequence, AuNPs tend to aggregate and form a low-density polymer network floating on the surface of the reaction medium, which is difficultly accessible to reactants. This fact decreases the catalytic capability of the system. On the contrary, after $\mathrm{CO}_{2}$ bubbling, 
water becomes a relatively good solvent for PDEAEMA: thanks to the formation of ammonium bicarbonates originated from the protonation of the amine groups by carbonic acid, the polymer chains switch from hydrophobic to hydrophilic and become more extended in solution. Hence, PDEAEMA-AuNPs become well dispersed in water, forming a homogeneous colloidal solution, in which all AuNPs are easily accessible to the reactants. This fact results in an enhancement of the reaction rate.

An alternative approach to regulating the catalytic activity of the system by controlling directly the reaction medium consists of the modification of the dimension of inhibitors. Grzybowski and co-workers [40] recently proposed AuNPs functionalized with $\mathrm{N}, \mathrm{N}, \mathrm{N}$-trimethyl(11-mercaptoundecyl) ammonium chloride (TMA) or 11-mercaptoundecanoic acid (MUA) for the catalysis of the reduction of 4-nitrophenol by $\mathrm{NaBH}_{4}$. AuNPs functionalized with TMA ligand are positively charged, while AuNPs functionalized with MUA ligand are characterized by a negative surface charge. The catalytic activity of both the types of AuNPs depends not only on the properties of the charged ligand shell, but also on the size of counterions surrounding the charged end-groups. In particular, the authors showed that large counterions can decrease or completely hinder the particle catalytic activity, while small counterions allow the reactant to reach the catalyst surface. Therefore, by exchanging the surrounding counterions, it is possible to dynamically switch between "on" and "off" active states. For example, they compare the catalytic activity of positively charged Au-TMA NPs combined with different counterions: $\mathrm{Cl}^{-}$(both from the TMA-Cl and TBA-Cl salts) and SDS ${ }^{-}$. As shown in Figure 6, the reaction can be catalyzed when the small $\mathrm{Cl}^{-}$counterion is considered, while when a bigger counterion (such as $\mathrm{SDS}^{-}$) is used, the reaction is stopped, since these ions behave as a barrier, hindering the diffusion of reactants toward the catalyst surface. A similar behavior is exhibited by Au-MUA NPs, even if in this case the catalytic activity is controlled by the size of positively charged counterions.
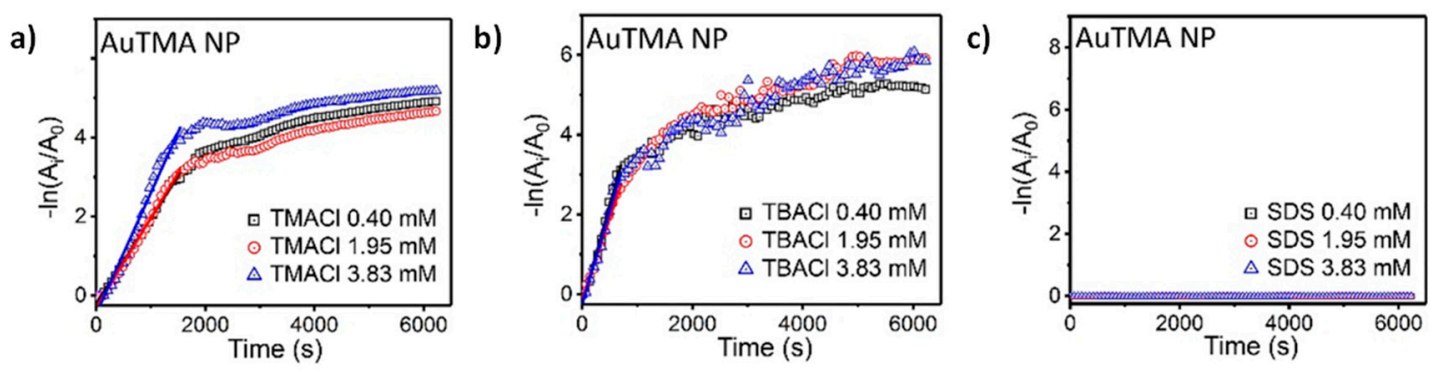

Figure 6. Kinetic plots for the 4-nitrophenol reduction catalyzed with AuTMA NPs in the presence of different counterions: (a) $\mathrm{Cl}^{-}$from $\mathrm{TMACl}$; (b) $\mathrm{Cl}^{-}$from $\mathrm{TBACl}$ and (c) $\mathrm{SDS}^{-}$at different concentrations. $A_{i}$ and $A_{0}$ are the values of the initial and instantaneous absorbance of 4-nitrophenol. Adapted and reprinted with permission from ref. [40]. Copyright (2018) American Chemical Society.

This study also shows how it is possible to perform a dynamic experiment controlling catalysis by switching counterions. Initially, Au-TMA NPs are surrounded by SDS ${ }^{-}$counterions and they do not show any catalytic activity. When an equivalent amount of CTAB is added to the reaction medium, the positively charged $\mathrm{CTA}^{+}$aggregate into micelles combining with negatively charged SDS $^{-}$. In this way big SDS ${ }^{-}$counterions are replaced by much smaller $\mathrm{Br}^{-}$coming from $\mathrm{CTAB}^{-}$and in these conditions, Au-TMA NPs become catalytically active. Catalysis can be again switched "off" by the addition of SDS and then "on" again by adding CTAB (Figure 7).

Reversible catalytic activity has also been obtained by modifying chemically the oxidation state of redox-responsive polymers. Elbert et al. [41] reported that it is possible to reversibly modulate the catalytic activity of Grubbs Second Generation catalyst for the ROMP (ring-opening metathesis polymerization) reaction of nerbornene when it is immobilized on the surface of $\mathrm{SiO}_{2} \mathrm{NPs}$ functionalized with poly (vinylferrocene). In fact, when poly(vinylferrocene) is oxidized, it increases its hydrophilicity and the polymeric network swells [42] creating a thicker shell layer that hinders the diffusion of apolar molecules (such as nerbornene) toward the catalyst. As a consequence, after 
oxidation of the poly(vinylferrocene), the ROMP reaction can be not catalyzed by the immobilized Grubbs Second Generation catalysts, which are not accessible to norbonene monomers. After the reduction of poly(vinylferrocene), the catalytic activity of the system can be restored.

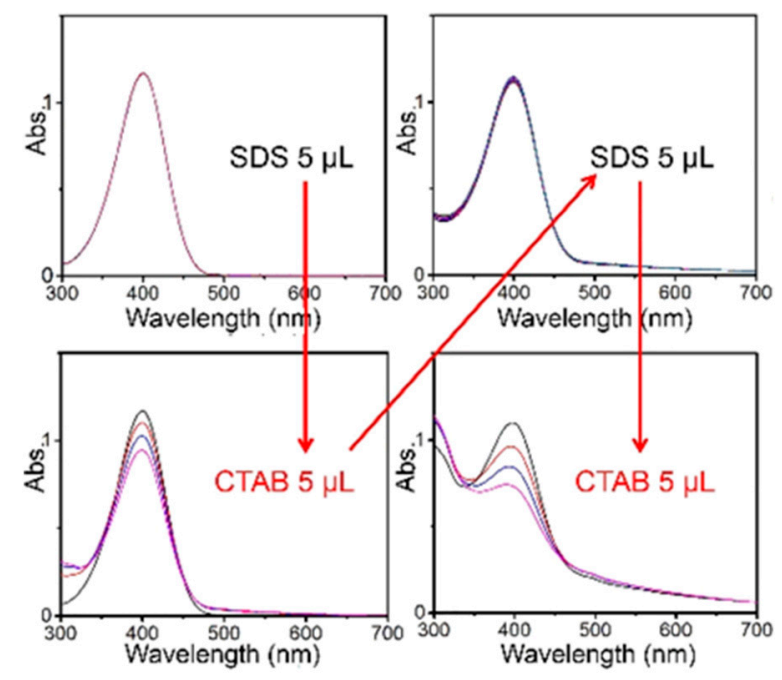

Figure 7. Changing of the catalytic activity of Au-TMA NPs by the consecutive addition of SDS or CTAB. Variations of UV-Vis spectra of 4-nitrophenol recorded over the time, during the sequence of SDS-CTAB-SDS-CTAB “steps". Reprinted with permission from ref. [40]. Copyright (2018) American Chemical Society.

\subsection{Light-Controlled Switchable Catalysis}

Light is particularly attractive as an alternative stimulus, as it can be delivered to nearly every place and at any time. It is noninvasive and can be precisely controlled with an appropriate source, offering excellent temporal and spatial resolution. Furthermore, using light of predetermined wavelengths allows the selective excitation and subsequent reactivity of specific molecular units [43].

Most of the photo-switchable systems reported in literature are based on the use of azobenzene units embedded in different matrices, the same happens in the field of responsive catalysis. Azobenzene is a photo-responsive molecule, which undergoes a trans-cis isomerization when it is illuminated with UV light and reverts back to the trans isomer when it is illuminated with visible light (or when it is heated). The isomerization entails an obvious variation of molecule geometry and volume, as well as modification of color and enhancement of dipole moment. All these properties have been widely exploited for the preparation of different types of smart materials, and it is possible to find in the literature interesting examples related to smart catalysis. Lawrence et al. [44] proposed the use of peptide-ligand-capped Au NPs with azobenzene units integrated into the biomolecular ligand for the switchable catalysis of the reduction of 4-nitrophenol by $\mathrm{NaBH}_{4}$. Illumination of the system with UV light causes the isomerization of the azobenzene units of the ligand from the trans to the cis form. This molecular switching process is propagated through the peptide structure on the nanoparticle surface and a global rearrangement of the ligand layer is obtained (Figure 8). These alterations of the molecular conformation at the AuNPs surface produce changes in the catalytic activity, since they can modify the number of the exposed active sites on the metal surface (trans configuration lead to a low exposition of the metal surface, while cis configuration enables an enhanced exposition of the metal surface toward the reactants). In particular, when azobenzene units are in the cis state, the peptide ligand shell is more open, and reactants can reach the catalyst surface more easily. In this condition, the reaction occurs quickly. On the contrary, when azobenzene moieties are in the trans configuration, the peptide chains collapse over the nanoparticle surface, hindering reactant diffusion and lowering the reaction rate. A new enhancement of the catalytic activity can be reached by simply illuminating the system with UV light. 


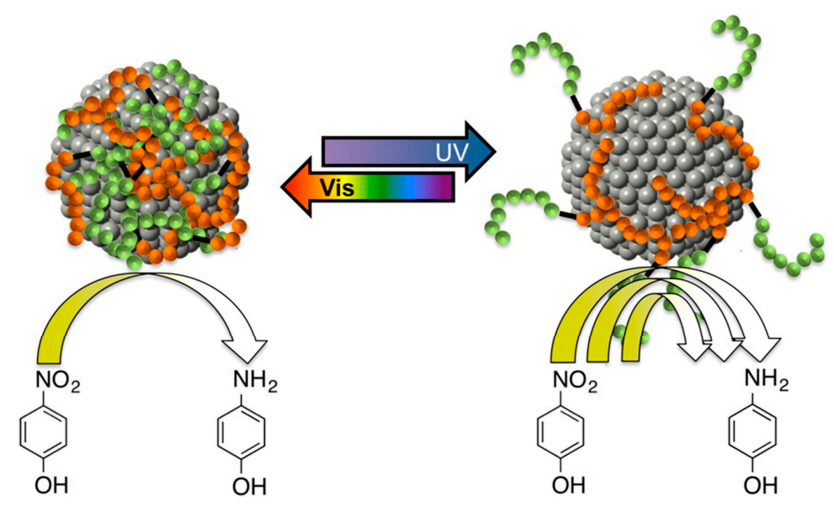

Figure 8. Schematic representation of the switchable behavior of peptide-ligand-capped Au NPs with azobenzene units (green portions) integrated into the biomolecular ligand under UV and visible illumination. Reprinted with permission from ref. [44]. Copyright (2016) American Chemical Society.

Another interesting approach was proposed by Szewczyk et al. [45]. The catalytic system is composed of gold nanoparticles whose surface has been functionalized with a ligand shell made of PEG chains alternated with PEG chains containing azobenzene units and a catalytic site (AzoPro) at the end (AzoPro50@AuNPs). In this case, the molecular catalyst is immobilized on catalytically idle nanoparticle surface and the considered reaction is the aldol reaction between $p$-nitrobenzaldehyde and cyclohexanone. When the system is illuminated with UV light, the azobenzene units inside the ligand layer switch to their cis form and undergo significant geometric restructuration. This fact leads to a modification of the catalyst (AzoPro) position and orientation in space, making it less accessible to reactants, which causes a significant decrease of the catalytic activity (Figure 9a). When the system is irradiated with visible light, the azobenzene units reisomerize into the trans form and the reaction rate can be enhanced again (Figure 9b). Actually, because of the need to reorganize the spatial disposition of the ligand shell, it is not possible to completely restore the fast kinetics achievable directly under visible illumination, thus, a non-totally switchable behavior can be obtained.

a)

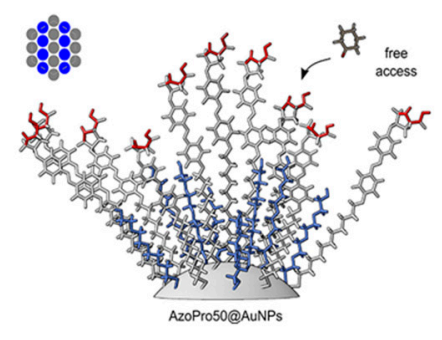

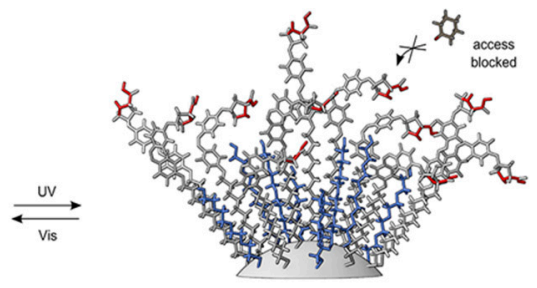

b)

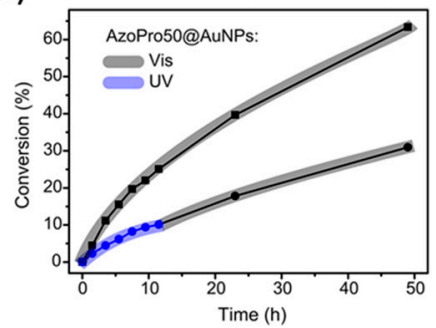

Figure 9. (a) Models of the ligand shells of AzoPro50@AuNPs under visible and UV light. Inset at the left shows the distribution of the ligands within the surface unit cells: PEG chains are in blue and PEG chains containing azobenzene units and AzoPro catalysts at the end are in grey. Accessibility of catalytic sites (marked in red) for substrate (cyclohexanone) is shown with curved arrays; (b) Influence of different types of illumination on the catalytic activity of AzoPro50@AuNPs during the aldol reaction of cyclohexanone with $p$-nitrobenzaldehyde. Adapted and reprinted with permission from ref. [45]. Copyright (2018) American Chemical Society.

Azobenzene derivatives (4,4'-diazene-1,2-diyldibenzoic acid, $\mathrm{H}_{2} \mathrm{AzDC}$ ) have recently been used to functionalize Metal-Organic Frameworks (MOF) and create a photoswitchable catalyst for the Knoevenagel condensation reaction [46]. The mechanism at the base of this photoswitchable behavior has been ascribed to the fact that $\mathrm{H}_{2} \mathrm{AzDC}$ located on the MOF pore wall isomerizes under UV illumination from the trans to the cis form, which is characterized by a higher steric hindrance. 
This isomerization causes a reduction of the volume in which reactants can diffuse, a decrease of the storage capacity of guest molecules inside the MOF and a modification of the capability of coordination of the catalyst (for example, according to DFT calculations, each trans- $\mathrm{H}_{2} \mathrm{AzDC}$ can be surrounded by 4 benzaldehyde molecules, while each cis- $\mathrm{H}_{2} \mathrm{AzDC}$ can accommodate only 3 benzaldehyde molecules). All these aspects lead to a drastic decrease of the reaction rate under UV illumination. Interestingly, the authors reported photoswitching not only of catalytic activity (high catalytic activity in dark, low catalytic activity under UV illumination), but also of catalytic selectivity (under UV illumination, the reaction occurs only with small reactants).

Light can be used as a stimulus to regulate the catalytic activity of the considered system to modify not only the ligands on catalysts, but also chemical species contained inside the reaction medium. Prins and co-workers [47] proposed a dynamic photo-switchable system composed of gold nanoparticles functionalized with a monolayer of C9-thiols terminated with a 1,4,7-triazacyclononane $(\mathrm{TACN}) \bullet \mathrm{Zn}^{2+}$ headgroup, which is able to catalyze the transphosphorylation of 2-hydroxypropyl-4nitrophenylphospate (HPNPP, a model substrate for RNA-hydrolysis). If a light-sensitive chemical substrate that is able to modify its affinity for the catalytic system upon photoisomerization is introduced inside the reaction medium, it is possible to use illumination to control the occurrence of the reaction. In this case, the stimulus does not have a direct effect on the catalyst, but on a molecule in the reaction medium, which acts as inhibitor. In the cited example, 4-(phenylazo)-benzoic acid, which combines a photoresponsive azobenzene and a carboxylic group negatively charged at $\mathrm{pH} 7$, was chosen as inhibitor. The trans isomer has a higher affinity for the considered catalyst in comparison to the cis one. In fact, the increase in polarity of azobenzene upon trans-cis isomerization reduces favorable hydrophobic interaction between 4-(phenylazo)-benzoic acid and the apolar part of the TACN monolayer. This means that when the inhibitor is in its trans form, it has a high affinity for the catalyst, to which it is bound thanks to hydrophobic interaction. This fact significantly reduces the efficiency of the catalyst. Upon illumination with UV light, it is possible to make 4-(phenylazo)-benzoic acid isomerize to its cis form, which has a low affinity for the catalyst. In this condition the inhibitor exerts a minor competition for the interaction with the catalyst and the reaction can be catalyzed more efficiently (Figure 10a). By illuminating the system with visible light again, it is possible to slow down the reaction and control the oscillations of the catalyst between states of high or low activity (Figure 10b).
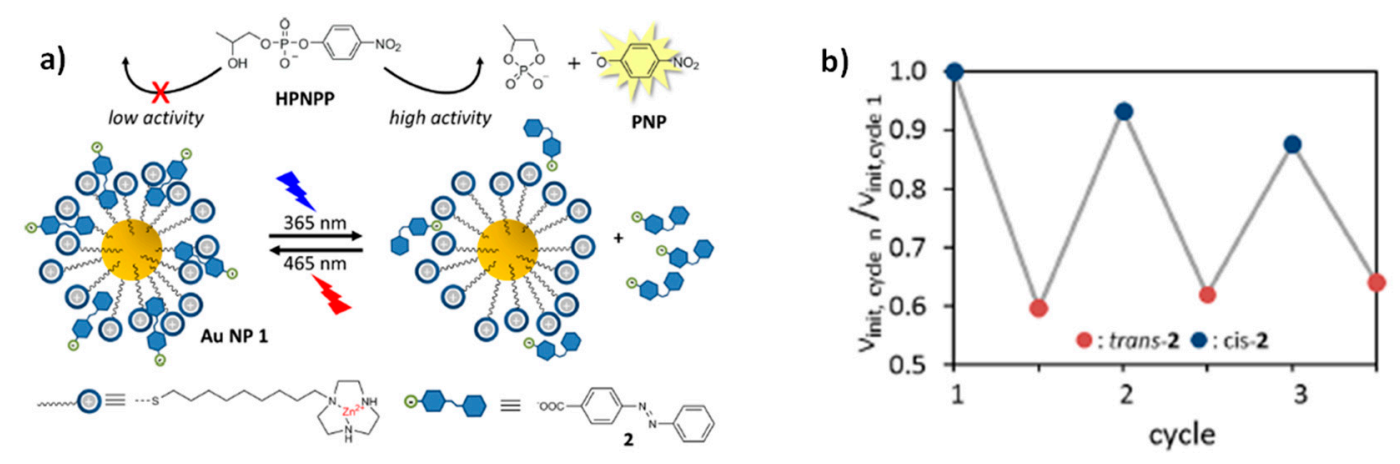

Figure 10. (a) Light-induced cis-trans isomerization of 4-(phenylazo)-benzoic acid changes its affinity toward the TACN. $\mathrm{Zn}^{2+}$ functionalized AuNPs, which catalyze the transphosphorylation of HPNPP; (b) plot of the initial rates (normalized on the initial rate of the first kinetics) for the reaction of transphosphorylation of HPNPP for several irradiation cycles. Adapted and reprinted with permission from ref. [47]. Copyright (2017) American Chemical Society.

Light can also be utilized to control the state of aggregation of the catalyst. Wei et al. [48] synthesized AuNPs functionalized with a mixture of "background" alkane amines and photoswitchable azobenzene-terminated alkane thiols that can be used for the catalysis of the hydrosilylation reaction of 4-methoxybanzaldeydhe. In the absence of light, the NPs remain unaggregated, exposing a 
large surface area that promotes the efficient catalysis of the reaction. Upon irradiation with UV light, the NPs aggregate, reducing the solvent-exposed surface area and effectively switching the catalysis off. The catalytic activity is regained upon visible irradiation and NP dispersion (Figure 11).

a)

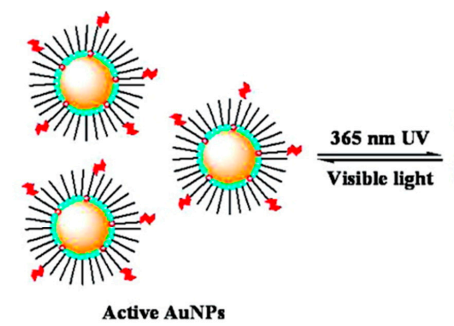

Active AuNPs

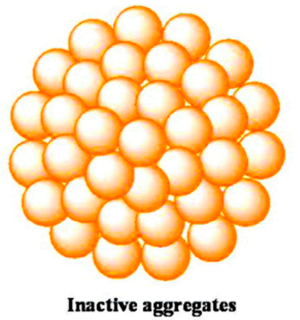

Inactive aggregates

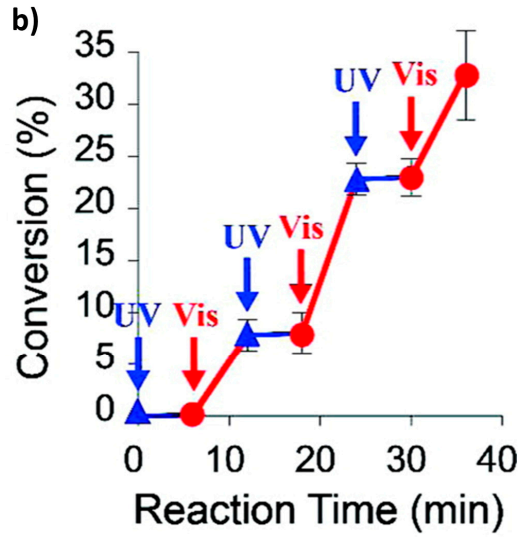

Figure 11. (a) Schematic representation of photoswitchable AuNPs: they are dispersed and catalytically active under visible illumination, while catalytically inactive when they are aggregated under UV illumination; (b) conversion efficiency (\%) for the AuNP-catalyzed hydrosilylation of 4-methoxybenzaldehyde as a function of time and as a function of irradiation type: visible light (red portions of the curve) and UV irradiation (blue portions). Adapted and reprinted with permission from ref. [48]. Copyright (2010) American Chemical Society.

The ability to switch catalysis "on" and "off" derives from light-stimulated reversible aggregation of the functionalized AuNPs. In particular, when AuNPs are illuminated with UV light, the azobenzene units isomerize from trans to cis configuration and develop electric dipoles. In non-polar solvent, like toluene (which is used in this reaction), these dipoles translate into attractive forces between NPs, leading to the formation of aggregates. After illumination with visible light, azobenzene units undergo a cis-trans isomerization and aggregates dissolve. The authors reported that it is possible to perform three subsequent catalytic cycles, but beyond this point not all NPs aggregate upon UV illumination and catalysis cannot be completely switched off.

On the contrary, Klajn and coworkers [49] reported that it is possible to enhance the reaction rate by inducing catalyst aggregation, by trapping reactants within dynamically self-assembled nanoflasks. The proposed catalytic system is composed of NPs $\left(\mathrm{Au}, \mathrm{Fe}_{3} \mathrm{O}_{4}\right.$ or $\left.\mathrm{SiO}_{2}\right)$ functionalized with azobenzene containing ligands. When suspended in apolar solvent (e.g., toluene) and illuminated with UV light, they self-assemble as a result of the attractive dipole-dipole interactions between cis-azobenzene moieties on different NPs. Supracrystals are formed: the NPs are closely packed, leaving small, essentially polar (cis-azobenzene rich) cavities, which are ideal for the trapping of small polar molecules contained inside the apolar solvent. If pairs of molecules that can potentially react with each other are simultaneously trapped, the confinement inside the cavities of the supracrystal accelerates the reaction between them. Then, once the product has formed, the aggregate can be disintegrated by visible light irradiation, the product can be released, recovered and the whole cycle can be repeated (Figure 12a). The acid-catalyzed hydrolysis of an acetal to its aldehyde in water-saturated toluene has been studied and it has been verified that in the presence AuNPs aggregate (formed after UV illumination) the reaction proceeds several time faster than in the absence of NPs or in presence of NPs but in absence of UV illumination (AuNPs are not aggregated) (Figure 12b). 
a)

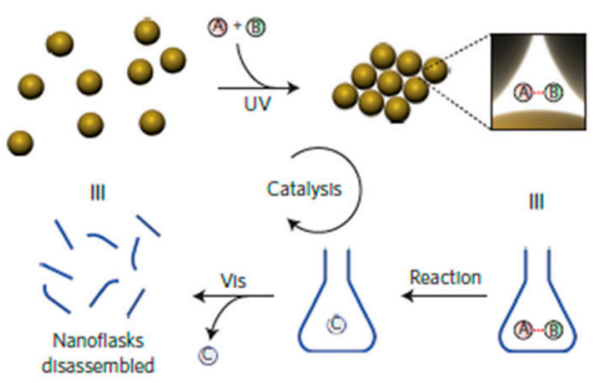

b)

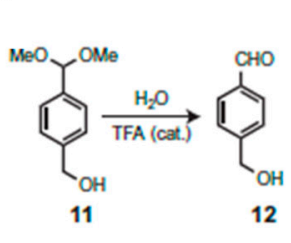

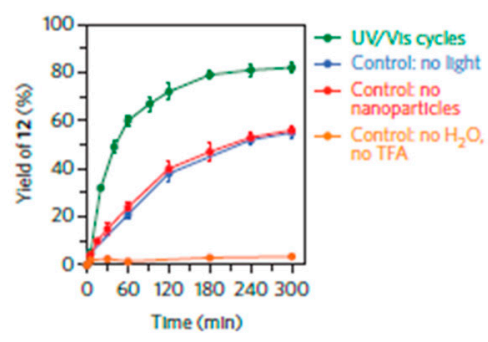

Figure 12. (a) Schematic representation of how the reversible formation of confined spaces can accelerate a chemical reaction; (b) reaction example: hydrolysis of acetal 11. Light-accelerated hydrolysis of $\mathbf{1 1}$ in different conditions. Reprinted with permission from ref. [49]. Copyright (2016) Nature Publishing Group.

Interestingly, the authors demonstrated that this trapping process can also alter the chemical reactivity of the guest species and the reaction stereoselectivity. For example, in the case of anthracene dimerization, if the reaction occurs in the absence of NP aggregates, almost exclusively the anti-product can be obtained; vice-versa, in the presence of nanoflasks, the syn-isomer is the main product. Probably, inside the NPs aggregates, anthracene pre-organizes itself via hydrogen-bonding with cis-azobenzene units of the NPs and this fact leads to a high yield of the otherwise unstable product. Therefore, this trapping strategy can be employed not just to accelerate a reaction, but also to modify its stereoselectivity. Furthermore, by functionalizing the NPs with azobenzene units and additional ligands, it could be possible to make the trapping selective for the recognition of particular chemical species or achieve also reaction enantioselectivity.

\section{Smart Catalysis by Means of Variation of the Nature of Active Catalytic Sites}

An alternative strategy to obtain smart catalysis is based on the insertion inside the catalytic system of switchable active sites, which are able to respond to an external stimulus and restore their catalytic activity after deactivation (Figure 1e). In this case, the external stimulus is able to change the nature of active sites (in most of the cases, oxidation state) at the catalyst surface rather than modifying its accessibility and it is used to regenerate them, conferring to the catalyst good recyclability and long-term activity. An example of the application of this strategy is the work of Mao et al. [50], in which an electrochemical stimulus (i.e., applied potential) was used to continuously manipulate the number of activated catalytic sites of a catalytic system made of a porous carbon fiber electrode conformally coated with poly(vinylferrocene), which is used as catalyst for the Michael addition of methyl vinyl ketone and ethyl-2-oxycyclopentane carboxylate. Ferrocenium (i.e., the oxidized form of ferrocene) catalyzes this reaction as a Lewis acid, whereas ferrocene has no catalytic activity. Therefore, by applying to the electrode a potential sufficiently high to make the oxidation of the poly(vinylferrocene) to occur, it is possible to catalyze the reaction of interest. On the contrary, when the applied potential is much lower than that of the redox ferrocenium/ferrocene couple, the catalytic sites are in the inactive reduced-form and catalysis does not occur. The applied potential can be continuously modulated, and it is possible to reach intermediate states in which the catalytic system is characterized by the presence of reduced inert sites and oxidized active sites simultaneously. In this way, intermediate values of reaction rates can be achieved (Figure 13). 


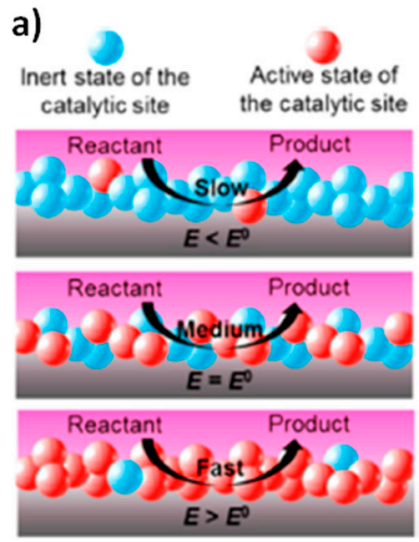

b)

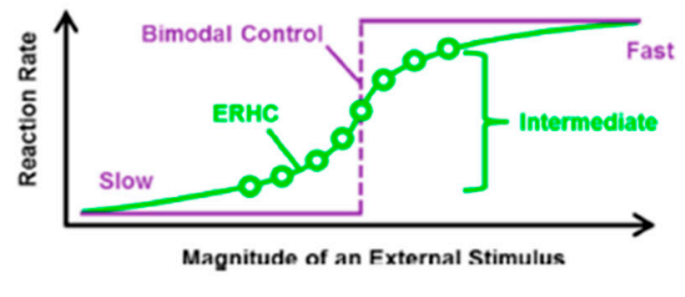

Figure 13. (a) Schematic illustration of the electrochemical control over the number of active sites on the surface of the porous carbon fiber electrode; (b) comparison between the electrochemically responsive heterogeneous catalyst (ERHC) and a "on/off" bimodal responsive catalyst for kinetic control. Reprinted with permission from ref. [50]. Copyright (2015) American Chemical Society.

Variations of the applied potential have also been used to dynamically control the nature of the products that can be obtained during a reduction reaction. Recently, Gao et al. [51] reported that during the electroreduction of $\mathrm{CO}_{2}$ it is possible to switch the type of obtained product (formate, hydrogen or $\mathrm{CO}$ ) by switching the applied potential using as catalyst carbon-supported Pd NPs. This is due to the fact that it is possible to modify the nature of the active sites inside the electrodes according to the applied potential, changing the affinity of the substrate towards different intermediate species and varying the possible reaction paths. A strategy based on variation of oxidizing conditions has been employed also to prepare smart catalysts based on metals supported on perovskites, characterized by a sort of self-healing behavior and with the capability to maintain a long-term catalytic activity, after the cyclic restoration of deactivated active sites. Many supported noble metal catalysts under prolonged operation show an irreversible loss of performance, which leads to their complete inactivation over the time. In fact, they suffer from particle sintering, coalescence, microstructural and morphological degradations. In parallel, poisoning due to the deposition of contaminants on the catalyst surface may occur. Overcoming these inconveniences is fundamental in order to achieve long-term stability. One of the proposed strategies is based on the stimuli-induced reintegration of the exsoluted catalytic metal phase inside the host matrix. Burnat et al. [52] reported that it is possible to regenerate a Ni-based catalytically active phase in SOFC anodes after the application of a chemical stimulus (in terms of oxygen partial pressure). The authors reported that under operation, the catalytically active Ni-phase segregates from the host perovskite matrix and the surface is poisoned by adsorbed $\mathrm{H}_{2} \mathrm{~S}$ and $\mathrm{C}$ species, leading to a lowering of its catalytic activity. Upon exposition to high oxygen partial pressure, microstructural self-regeneration takes place: the surface is cleaned off by the oxidation of poisoning species (sulfur species to $\mathrm{SO}_{2}$ and $\mathrm{C}$ to $\mathrm{CO}_{2}$ ) and $\mathrm{Ni}$ active sites are re-incorporated into the host perovskite lattice. Under operation, exsolution of the Ni phase and poisoning occur again, but the catalytic activity can be restored by further oxidation. Similar behavior has been previously reported by Nishihata et al. [53] in the case of Pd-perovskite catalyst used as converters to reduce the amount of nitrogen oxides, carbon monoxide and unburned hydrocarbons in automotive emission. Coarsened $\mathrm{Pd}$ particles can be redispersed under oxidizing conditions by reaction with a perovskite support (which works as a protective material), while they are exsoluted under reducing conditions. This concept has been demonstrated to be applicable with various transition metals on different perovskite oxide [54-56], and resulted in being particularly effective when high-surface area perovskite supports were employed, as demonstrated by Fornasiero and co-workers in different seminal papers [57]. Even if in these cases the external stimulus (high oxygen partial pressure) is used just to recover the lost 
catalytic activity, the same strategy (cycling between oxidizing ad reducing conditions) could be exploited in future to dynamically control the catalytic activity of the whole system.

In general, Pd-based catalysts are particularly suitable for integration into smart catalytic systems. Schlögl and co-workers demonstrated in pioneering works that the activity of Pd catalysts in alkyne-to-alkene hydrogenation is strongly influenced by the presence of subsurface carbon and hydrogen species $[58,59]$. For example, they showed that hydrogenation can be made either selective or unselective by controlling the amount of carbon incorporated in the Pd particles, which, in turn, can be regulated by the $\mathrm{H}_{2} / \mathrm{C}_{\mathrm{x}}$ ratio of the species that are present in the catalytic feed. In this scenario, C-uptake in Pd particles proceeds through the fragmentation of alkyne molecules at the beginning of the hydrogenation process. As a result of $C$ incorporation, hydrogen transport is inhibited, and hydrogen located in the subsurface layers of $\mathrm{Pd}$, which is normally involved in these types of catalytic processes (e.g., the hydrogenation of an alkene into the corresponding alkane), cannot effectively participate to hydrogenation. This allows the alkyne hydrogenation to be selectively limited to the production of alkene, without any further extension to alkane species. Interestingly, the authors also pointed out the possibility to induce fluctuations in selectivity, by exploiting the kinetic mismatch among $\mathrm{H}$ subsurface diffusion, $\mathrm{C}$-uptake and surface reaction rates. If the population of the catalyst subsurface undergoes significant changes during the reaction, spontaneous switching between selective and unselective hydrogenation regimes can be observed. In addition to being milestones in research on Pd-based catalysts, as they demonstrated the intimate mechanisms of hydrogenation reaction, unraveling the role of subsurface layers, these works can also a major source of inspiration for the design of a new generation of smart catalysts, in which catalytic feed becomes a tool to control the overall process.

\section{Macroscopic Smart Catalytic Systems}

Systems that are able to modify their catalytic activity in response to the application of an external stimulus by altering their configuration on a macroscopic scale offer the opportunity to find alternative catalytic strategies in comparison to the traditional dispersion of heterogeneous catalysts in solution. In the literature, it is possible to find just a few examples, but they are extremely interesting, given that they pave the way to the preparation of complex dynamic catalytic devices. For example, Niazov et al. [60] proposed the preparation of photo-switchable electrode for the electrocatalytic reduction of $\mathrm{H}_{2} \mathrm{O}_{2}$. The system is composed of an indium tin oxide (ITO) electrode functionalized with a photo-isomerizable monolayer of nitro-spiropyran (SP) derivatives immersed in a solution containing citrate-functionalized Pt NPs. The nitro-spiropyran monolayer lacks affinity for the negatively charged $\mathrm{Pt}$ NPs, which are well distributed inside the solution; as a consequence, the functionalized-ITO electrode is catalytically inactive toward the $\mathrm{H}_{2} \mathrm{O}_{2}$ reduction. Upon illumination with UV light, nitro-spiropyran isomerizes to the nitro-merocyanine photoisomer, which is protonated in an acidic environment, leading to the formation of positively charged protonated nitro merocyanine $\left(\mathrm{MRH}^{+}\right)$. The resulting positively charged monolayer attracts the Pt NPs from the solution, enabling the formation of a NP-modified electrode. This system efficiently catalyzed the electrochemical reduction of $\mathrm{H}_{2} \mathrm{O}_{2}$. It is possible to convert the $\mathrm{MRH}^{+}$-monolayer to SP-monolayer by visible light irradiation, with consequent detachment of the Pt NPs and formation of a catalytically inactive electrode. By cyclic illumination and photoisomerization of the monolayer, the ITO electrode can be reversibly switched between an "on" and "off" state (for, at least, 10 cycles) (Figure 14). 
a)

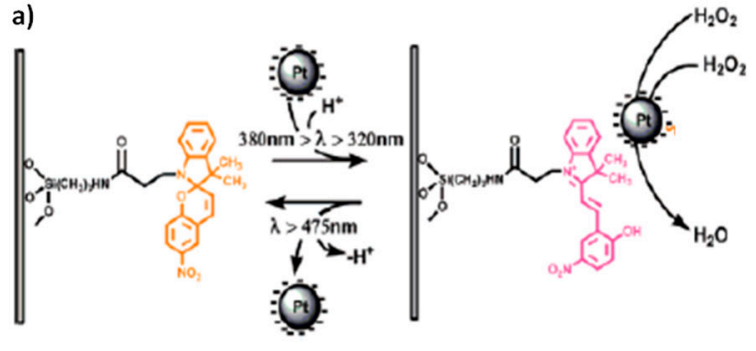

b)

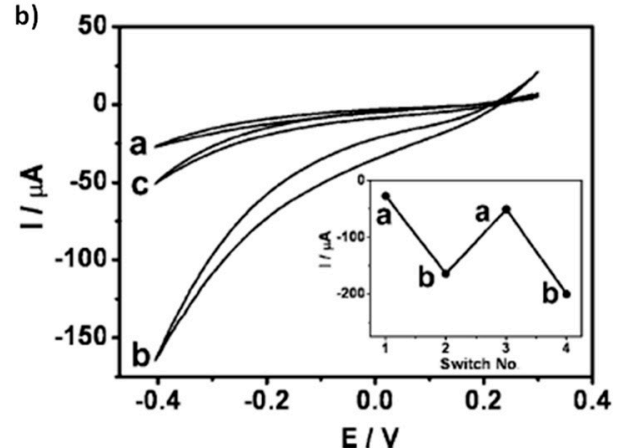

Figure 14. (a) Scheme of the photoswitchable behavior of the functionalized-ITO electrode under UV and visible illumination; (b) cyclic voltammograms corresponding to (a) the SP monolayer-modified ITO electrode treated with $\mathrm{Pt} \mathrm{NP}$ in the presence of $\mathrm{H}_{2} \mathrm{O}_{2}$, (b) the $\mathrm{MRH}^{+}$monolayer-functionalized electrode in the presence of Pt NPs and $\mathrm{H}_{2} \mathrm{O}_{2}$, and (c) back photoisomerization of the monolayer to the SP state and treatment of the system with $\mathrm{Pt} N P s$ and $\mathrm{H}_{2} \mathrm{O}_{2}$. The inset shows photoswitchable amperometric responses of the photoisomerizable electrode treated with Pt NPs $/ \mathrm{H}_{2} \mathrm{O}_{2}$ in the presence of (a) the SP state and (b) the $\mathrm{MRH}^{+}$state. Adapted and reprinted with permission from ref. [60]. Copyright (2007) American Chemical Society.

The same reaction can be dynamically catalyzed by a Pt wire (length $2 \mathrm{~cm}$, diameter $250 \mu \mathrm{m}$ ) functionalized with a tightly packed self-assembled monolayer (SAM) of 1-naphthalenethiol [61]. In this system, the control of the switching between catalytically active and inactive state is obtained by means of elastic deformation of the metal wire. Initially, the SAM-passivated Pt wire is catalytically inactive toward $\mathrm{H}_{2} \mathrm{O}_{2}$ present in the surrounding medium, since there is no direct contact between the catalyst surface and its substrate. However, when the wire is slightly bent, the SAM becomes less compact in the bending regions, allowing an increased access of the reagents to the metal catalytic surface (Figure 15a). This fact is reflected in the Pt-catalyzed decomposition of $\mathrm{H}_{2} \mathrm{O}_{2}$, producing $\mathrm{O}_{2}$ bubbles. When the compressive force is removed and the wire unbends, the protective SAM "heals" and catalysis is interrupted (Figure 15b). Upon a further bending, it is possible to reactivate the catalysis. Anyway, the bending has not to be very pronounced, otherwise the SAM is permanently damaged and the catalysis cannot be stop even if the wire restores its initial shape.

a)

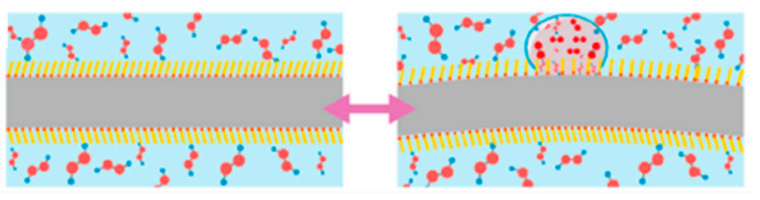

b)

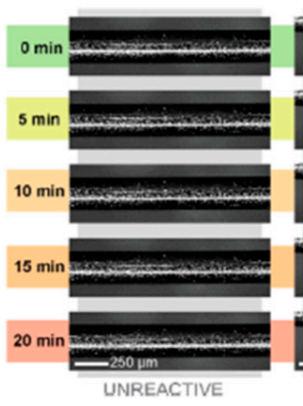

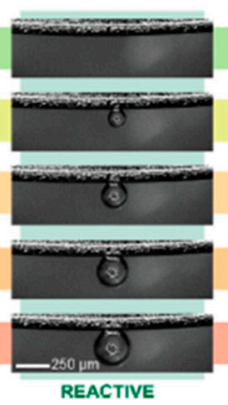

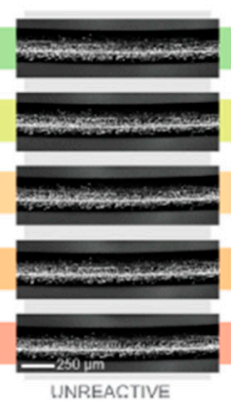

Figure 15. (a) Schematic representation of the SAM modification on the Pt wire after mechanical deformation; (b) experimental images of the Pt rod covered with the SAM of 1-naphthalenethiol, immersed in the $0.5 \% \mathrm{H}_{2} \mathrm{O}_{2}$ solution. In the initial state (left), the rod is unreactive; bending of the rod (middle) exposes the catalytic surface and initiates a reaction, which manifests itself by the formation of oxygen bubbles; when the compressive force is removed (right) the rod unbends, and catalysis ceases. Adapted and reprinted with permission from ref. [61]. Copyright (2017) American Chemical Society.

Another interesting system has been proposed by Aizenberg and co-workers [62]: a device composed of a $\mathrm{pH}$ - or temperature-responsive hydrogel on which tubular microstructures are 
supported. In addition, the tips of these microstructures are functionalized with a catalyst. In response to an external stimulus (variation of $\mathrm{pH}$ or temperature), reconfiguration of the hydrogel (swelling and de-swelling) occurs, inducing reversible actuation of microstructures into and out of a nutrient layer, where reactants are contained. This actuation serves as a precise on/off switch for catalysis of different chemical reactions, according to the catalyst nature. The hydrogel can be made of poly(acrylamide-co-acrylic acid), which is a $\mathrm{pH}$-sensitive polymer (swelling when $\mathrm{pH}$ is between 5 and 10 and shrinking when $\mathrm{pH}$ is out of these limits), or PNIPAm (swelling when $\mathrm{T}$ is lower than $32{ }^{\circ} \mathrm{C}$ and shrinking when $\mathrm{T}>32{ }^{\circ} \mathrm{C}$ ). If the system is soaked inside a reaction medium, when the hydrogel is swollen, the embedded microstructures straighten and their catalyst-functionalized tips enter the reagent layer, triggering the reaction of interest. When the hydrogel shrinks, microstructures bend, and they are removed from the reagent layer, interrupting the reaction. According to this sequence, in the case of poly(acryl amide-co-acrilic acid), a chemical stimulus ( $\mathrm{pH}$ variation) is converted into a mechanical action which leads to a chemical reaction, realizing a synchronized cascade of chemomechanical energy interconversions $\left(C_{1} \rightarrow M \rightarrow C_{2}\right)$. The operation has been tested through different processes: if fluorescein is used as a marker, it is possible to intermittently quench its fluorescence by inducing the contact with potassium iodide in the nutrient layer; if $\mathrm{Pt}$ nanoparticles are immobilized on the microstructure tips and $\mathrm{H}_{2} \mathrm{O}_{2}$ is introduced in the nutrient layer, $\mathrm{H}_{2} \mathrm{O}_{2}$ reduction and $\mathrm{O}_{2}$ production can be dynamically catalyzed. Furthermore, the authors showed that when a temperature-responsive hydrogel is considered and the system is coupled with exothermic catalytic reactions it is possible to create self-powered, self-regulated oscillating systems. When $\mathrm{T}$ is lower than the hydrogel LCST, the microstructures are straight, and their catalyst-functionalized tips are in contact with the reagent layer, triggering the occurrence of the exothermic reaction. When temperature increases, as a result of the chemically generated heat, and exceeds LCST, contraction of the hydrogel is triggered, removing the catalyst and the microstructures from the reagent layers. The exothermic reaction is stopped and temperature gradually falls, until it reaches T $<$ LCST. At this point, the cycle restarts, giving rise to continuous, self-regulated $\mathrm{C} \leftrightarrow \mathrm{M}$ oscillations (Figure 16).

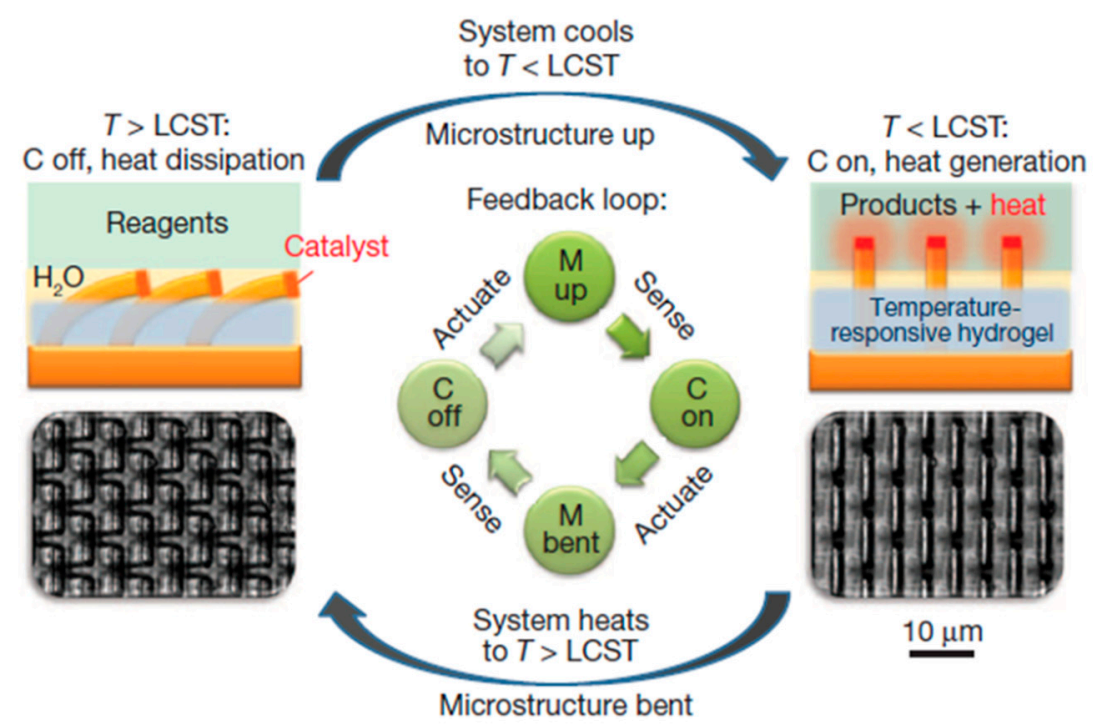

Figure 16. Schematic of the temperature-regulating catalytic system showing a $C \leftrightarrow M$ feedback loop, in which mechanical action of temperature-responsive gel is coupled with an exothermic reaction. The side-view schematic and top-view microscope images depict on/off states of the reaction in the top layer. Reprinted with permission from ref. [62]. Copyright (2012) Nature Publishing Group.

Another alternative switchable device that can be used for catalytic purposes is represented by the so-called "phactalysts" [63]. These are bimorph photo-actuators obtained by combining materials characterized by very different coefficients of thermal expansion (CTEs), such as a membrane filter 
made of polycarbonate (PC) and single wall carbon-nanotubes (SWNTs), and illuminating it with Vis/NIR, light. Also, in this case, an external stimulus (light) is used to introduce a deformation in the device, which is in turn exploited to regulate the occurrence of a chemical reaction with precise spatial and temporal control. In particular, it is exploited the capability of SWNTs to absorb light in the Vis/NIR range and convert it into heating that is transferred to the under-layer made of polycarbonate. Since the two involved materials are characterized by very different CTEs, during illumination, the underlying PC substrate is significantly more expanded than the upper-layer of SWNTs, and the final result is a structure which curls in the direction of light. On the contrary, the whole device maintains a flat configuration in dark or under UV illumination. If reactants involved in a chemical reaction are dropped on the two opposite bending rims of the device, illumination can be used to put them in contact and trigger the reaction (Figure 17a). This strategy is particularly useful in the case of dangerous reactions (e.g., flammable or explosives reactants or products) and when we want to minimize the operator participation for safety concerns. The strongly exothermic redox reaction between glycerol and permanganate has been considered as an example (Figure 17b). Furthermore, this study demonstrates that it is possible to confer to the device a proper photo-catalytic activity by blending $\mathrm{TiO}_{2} \mathrm{NPs}$ within the SWNT network. In this case, illumination is used to control the occurrence and stopping of photochemical reactions catalyzed by $\mathrm{TiO}_{2} \mathrm{NPs}$ (Figure 17c). As an example, the degradation of methylene blue (MB), photocatalyzed by $\mathrm{TiO}_{2} \mathrm{NPs}$ under Vis-UV illumination, was considered. In this case, a few droplets of MB solution were located at the center of the "phactalyst". When the whole system is illuminated with Vis/NIR light, it curls, and the bending rims cover the $\mathrm{MB}$ solution, preventing its direct illumination. As a result, no photo-degradation occurs. On the other hand, when UV light is used for illumination, the phactalyst maintains its flat configuration and UV light can be employed to promote the MB photodegradatrion. By illuminating the system with Vis-NIR light, the reaction can be stopped in any moment (Figure 17d,e).

a)

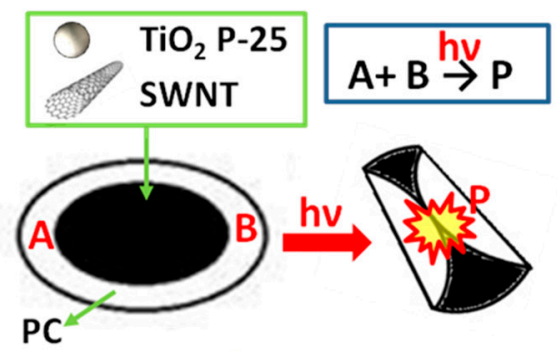

b) $14 \mathrm{KMnO}_{4}+4 \mathrm{C}_{3} \mathrm{H}_{5}(\mathrm{OH})_{3} \stackrel{\mathrm{H}^{+}}{\longrightarrow} 7 \mathrm{~K}_{2} \mathrm{CO}_{3}+7 \mathrm{Mn}_{2} \mathrm{O}_{3}+5 \mathrm{CO}_{2}+16 \mathrm{H}_{2} \mathrm{O}$
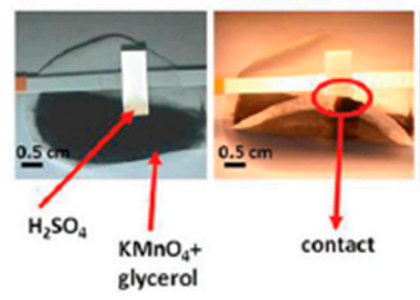

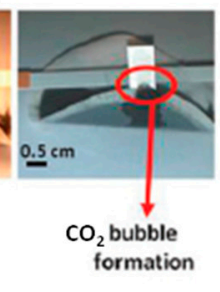

c)

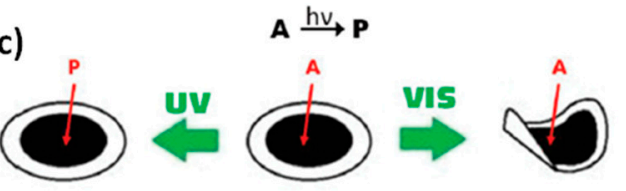

d)

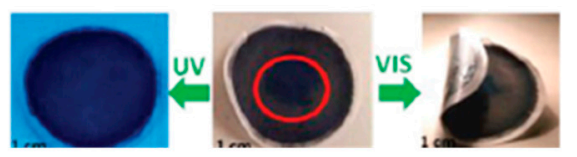

e)

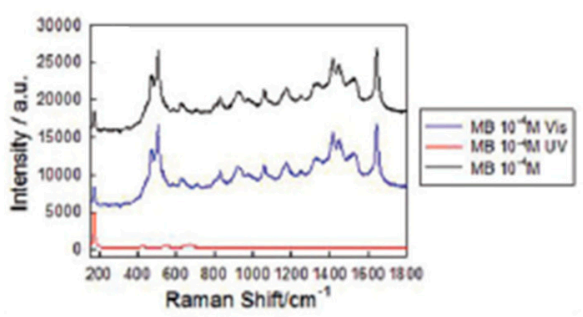

Figure 17. (a) Scheme of the use of a "phactalyst" in light-driven remote triggering of chemical reactions; (b) snapshots illustrating how the "phactalyst" can be exploited to trigger the redox reaction between potassium permanganate, glycerol and sulfuric acid; (c) scheme of the use of a "phactalyst" as optical shutter: a photochemical reaction can be either activated (UV irradiation) or prevented (Vis irradiation); (d) proof-of-concept experiment: optical images showing a droplet of $\mathrm{MB} 10^{-4} \mathrm{M}$ solution located at the center of a "phactalyst" under UV (left), dark (center) and Vis/NIR illumination (right); (e) Raman spectra of the MB suspension before (black line) and after either Vis/NIR (blue line) or UV (red line) illumination. Adapted and reprinted with permission from ref. [63]. Copyright (2017) Royal Society of Chemistry. 


\section{Conclusions and Outlook}

The use of the concepts and materials at the basis of stimuli-responsive systems enables the preparation of a new class of catalysts characterized by switchability (they can pass, on demand, from an "on" active state to an "off" inactive state), adaptability (they are able to modulate dynamically their catalytic efficiency, according to the properties of the surrounding) and high recyclability (they can be easily separated from the reaction medium after their conversion into their inactive state and can be reused after their reconversion in their active configuration). A truly dynamic control over the catalytic properties of these systems is generally pursued by following a few different approaches: the most widespread strategy relies on regulating either the diffusion dynamics of chemical species toward the catalyst surface or the aggregation state of the catalyst or both. Recent alternatives rely on the insertion of active sites inside the catalytic system, which are able to modify their nature according to the external environment. A complete investigation of dynamic systems based on variable diffusion of inhibitors is still lacking, while no examples of switchable diffusion of activators have been reported so far. Both the diffusion and aggregation strategies are effective to obtain a precise temporal control (on/off) of a chemical reaction, but switchable aggregation does not allow modulation of the reaction rate in a continuous way or regulation of the quantity of the catalytic activity. On the other hand, control of the catalyst aggregation yields systems characterized by higher recyclability, leading to systems that are able to fully preserve their catalytic efficiency over different cycles. The dynamic modification of the active catalytic site is particularly useful in the case of redox reactions, in particular for systems that require long-term stability and high recyclability. Temperature is still the most frequently employed stimulus, and thermo-responsive polymers functionalized with catalytic units are the most investigated systems. The large majority of the examples are based on PNIPAm, but recently, new polymers with different transition temperatures have been developed, leading to the preparation of a palette of catalytic systems whose efficiency can be switched in a wide range of temperatures $\left(20-50^{\circ} \mathrm{C}\right)$. Light offers interesting opportunities as an alternative stimulus: excellent spatial and temporal resolution, easy tunability and selective excitation of specific molecular units. Also, in this case, most of the studies are based on similar systems (azobenzene derivatives), but a larger variability could be obtained by employing different photo-sensible units (for example, spiropyran, stilbene, diarylethene). Further study of alternative stimuli such as variations of chemical composition of the surrounding medium (at the moment current studies are limited to variations of $\mathrm{pH}$ or ionic strength), electric and magnetic field (whose employment, in most of the cases, is limited to obtain an easy separation of catalysts after the end of their work $[64,65]$ or to the induction of heating [66] for endothermic reactions) or mechanical stress (also thanks to the exploitation of piezoelectric effect for the generation of catalytically active species [67]) would be desirable, paving the way to the preparation of dynamic systems based on new and unconventional strategies. The preparation of macroscopic catalytic devices has already opened the way in this direction, finding alternative strategies in comparison to the traditional dispersion of heterogeneous catalysts in solution, which also enabled the synergistic combination of precise temporal and spatial control of chemical reactions. However, most of these works are still limited to the study of simple model reactions (in particular, the reduction of 4-nitrophenol to 4-aminophenol by $\mathrm{NaBH}_{4}$ ), whereas the application of these systems to other, more technologically relevant reactions is still completely unexplored. For example, dynamic catalytic systems could find interesting applications in production of solar fuels or energy vectors from renewable sources. The incorporation of all these properties inside artificial heterogeneous catalysts is a fundamental step forward in the preparation of nature-inspired catalytic systems. Among the future challenges, we can envision the possibility of realizing systems that are able to control the stereo-selectivity of product formation in a dynamic way, and to activate themselves only after the selective recognition of chemical species inside a complex mixture of different substrates. This will open the door to the development of multi-responsive systems controlled by chemical reactions. 
Author Contributions: Conceptualization and Writing-Original Draft Preparation, I.V., Writing-Review and Editing: I.V. and I.A.

Funding: This research received no external funding.

Conflicts of Interest: The authors declare no conflict of interest.

\section{References}

1. Grzybowski, B.A.; Huck, W.T.S. The nanotechnology of life-inspired systems. Nat. Nanotechnol. 2016, 11, 585-592. [CrossRef] [PubMed]

2. Zhai, L. Stimuli-responsive polymer films. Chem. Soc. Rev. 2013, 42, 7148-7160. [CrossRef] [PubMed]

3. Dai, S.; Ravi, P.; Tam, K.C. pH-Responsive polymers: Synthesis, properties and applications. Soft Matter 2008, 4, 435-449. [CrossRef]

4. Jochum, F.D.; Theato, P. Temperature- and light-responsive smart polymer materials. Chem. Soc. Rev. 2013, 42, 7468-7483. [CrossRef] [PubMed]

5. Manouras, T.; Vamvakaki, M. Field responsive materials: Photo-, electro-, magnetic- and ultrasound-sensitive polymers. Polym. Chem. 2017, 8, 74-96. [CrossRef]

6. Thévenot, J.; Oliveira, H.; Sandre, O.; Lecommandoux, S. Magnetic responsive polymer composite materials. Chem. Soc. Rev. 2013, 42, 7099-7116. [CrossRef] [PubMed]

7. Davis, D.A.; Hamilton, A.; Yang, J.; Cremar, L.D.; Van Gough, D.; Potisek, S.L.; Ong, M.T.; Braun, P.V.; Martínez, T.J.; White, S.R.; et al. Force-induced activation of covalent bonds in mechanoresponsive polymeric materials. Nature 2009, 459, 68-72. [CrossRef] [PubMed]

8. Stuart, M.A.C.; Huck, W.T.S.; Genzer, J.; Müller, M.; Ober, C.; Stamm, M.; Sukhorukov, G.B.; Szleifer, I.; Tsukruk, V.V.; Urban, M.; et al. Emerging applications of stimuli-responsive polymer materials. Nat. Mater. 2010, 9, 101-113. [CrossRef] [PubMed]

9. Wei, M.; Gao, Y.; Li, X.; Serpe, M.J. Stimuli-responsive polymers and their applications. Polym. Chem. 2017, 8, 127-143. [CrossRef]

10. Schmaljohann, D. Thermo- and pH-responsive polymers in drug delivery. Adv. Drug Deliv. Rev. 2006, 58, 1655-1670. [CrossRef] [PubMed]

11. Hager, B.M.D.; Greil, P.; Leyens, C.; Zwaag, S.; Van Der Schubert, U.S. Self-healing Materials. Adv. Mater. 2010, 5424-5430. [CrossRef] [PubMed]

12. Hu, J.; Liu, S. Responsive polymers for detection and sensing applications: Current status and future developments. Macromolecules 2010, 43, 8315-8330. [CrossRef]

13. Ionov, L. Hydrogel-based actuators: Possibilities and limitations. Mater. Today 2014, 17, 494-503. [CrossRef]

14. Vassalini, I.; Alessandri, I. Spatial and Temporal Control of Information Storage in Cellulose by Chemically Activated Oscillations. ACS Appl. Mater. Interfaces 2015, 7, 28708-28713. [CrossRef] [PubMed]

15. Stoll, R.S.; Hecht, S. Artificial light-gated catalyst systems. Angew. Chem. Int. Ed. 2010, 49, 5054-5075. [CrossRef] [PubMed]

16. Blanco, V.; Leigh, D.A.; Marcos, V. Artificial switchable catalysts. Chem. Soc. Rev. 2015, 44, 5341-5370. [CrossRef] [PubMed]

17. Lüning, U. Switchable catalysis. Angew. Chem. Int. Ed. 2012, 51, 8163-8165. [CrossRef] [PubMed]

18. Choudhury, J. Recent developments on artificial switchable catalysis. Tetrahedron Lett. 2018, 59, 487-495. [CrossRef]

19. Heskins, M.; Guillet, J.E. Solution Properties of Poly(N-isopropylacrylamide). J. Macromol. Sci. A 1968, 2, 1441-1455. [CrossRef]

20. Lu, Y.; Mei, Y.; Drechsler, M.; Ballauff, M. Thermosensitive core-shell particles as carriers for Ag nanoparticles: Modulating the catalytic activity by a phase transition in networks. Angew. Chem. Int. Ed. 2006, 45, 813-816. [CrossRef] [PubMed]

21. Jiang, X.; Xiong, S.A.; An, Y.; Zheng, P.; Zhang, W.; Shi, L. Thermoresponsive Hydrogel of Poly(glycidylmethacrylate-co-N-isopropylacrylamide) as a Nanoreactor of Gold Nanoparticles. J. Polym. Sci. A Polym. Chem. 2007, 45, 2812-2819. [CrossRef]

22. Wang, Y.; Wei, G.; Wen, F.; Zhang, X.; Zhang, W.; Shi, L. Synthesis of gold nanoparticles stabilized with poly/ $\mathrm{N}$-isopropylacrylamide)-co-poly(4-vinyl pyridine) colloid and their application in responsive catalysis. J. Mol. Catal. A Chem. 2008, 280, 1-6. [CrossRef] 
23. Xiong, D.; Li, Z.; Zou, L.; Zhenping, H.; Liu, Y.; An, Y.; Rujiang, M.; Shi, L. Modulating the catalytic activity of $\mathrm{Au} /$ micelles by tunable hydrophilic channels. J. Colloid Interface Sci. 2010, 341, 273-279. [CrossRef] [PubMed]

24. Huang, X.; Yin, Y.; Tang, Y.; Bai, X.; Zhang, Z.; Xu, J.; Liu, J.; Shen, J. Smart microgel catalyst with modulatory glutathione peroxidase activity. Soft Matter 2009, 5, 1905-1911. [CrossRef]

25. Liu, X.Y.; Cheng, F.; Liu, Y.; Liu, H.J.; Chen, Y. Preparation and characterization of novel thermoresponsive gold nanoparticles and their responsive catalysis properties. J. Mater. Chem. 2010, 20, 360-368. [CrossRef]

26. Li, S.; Ge, Y.; Tiwari, A.; Cao, S. A temperature-responsive nanoreactor. Small 2010, 6, 2453-2459. [CrossRef] [PubMed]

27. Borrmann, R.; Palchyk, V.; Pich, A.; Rueping, M. Reversible Switching and Recycling of Adaptable Organic Microgel Catalysts (Microgelzymes) for Asymmetric Organocatalytic Desymmetrization. ACS Catal. 2018, 8 , 7991-7996. [CrossRef]

28. Xiao, C.; Chen, S.; Zhang, L.; Zhou, S.; Wu, W. One-pot synthesis of responsive catalytic Au@PVP hybrid nanogels. Chem. Commun. 2012, 48, 11751-11753. [CrossRef] [PubMed]

29. Fedorczyk, A.; Ratajczak, J.; Kuzmych, O.; Skompska, M. Kinetic studies of catalytic reduction of 4-nitrophenol with $\mathrm{NaBH}_{4}$ by means of Au nanoparticles dispersed in a conducting polymer matrix. J. Solid State Electrochem. 2015, 19, 2849-2858. [CrossRef]

30. Li, G.L.; Tai, C.A.; Neoh, K.G.; Kang, E.T.; Yang, X. Hybrid nanorattles of metal core and stimuli-responsive polymer shell for confined catalytic reactions. Polym. Chem. 2011, 2, 1368-1374. [CrossRef]

31. Zhang, J.; Zhang, W.; Wang, Y.; Zhang, M. Palladium-iminodiacetic acid immobilized on $\mathrm{pH}$-responsive polymeric microspheres: Efficient quasi-homogeneous catalyst for Suzuki and Heck reactions in aqueous solution. Adv. Synth. Catal. 2008, 350, 2065-2076. [CrossRef]

32. Zhang, M.; Zhang, W. Pd nanoparticles immobilized on pH-responsive and chelating nanospheres as an efficient and recyclable catalyst for suzuki reaction in water. J. Phys. Chem. C 2008, 112, 6245-6252. [CrossRef]

33. Monai, M.; Montini, T.; Fonda, E.; Crosera, M.; Delgado, J.J.; Adami, G.; Fornasiero, P. Nanostructured Pd-Pt nanoparticles: Evidences of structure/performance relations in catalytic H2production reactions. Appl. Catal. $B$ Environ. 2018, 236, 88-98. [CrossRef]

34. Zheng, P.; Zhang, W. Synthesis of efficient and reusable palladium catalyst supported on $\mathrm{pH}$-responsive colloid and its application to Suzuki and Heck reactions in water. J. Catal. 2007, 250, 324-330. [CrossRef]

35. Wang, Y.; Zhang, J.; Zhang, W.; Zhang, M. Pd-catalyzed C-C cross-coupling reactions within a thermoresponsive and $\mathrm{pH}$-responsive and chelating polymeric hydrogel. J. Org. Chem. 2009, 74, 1923-1931. [CrossRef] [PubMed]

36. Wang, Y.; Yan, R.; Zhang, J.; Zhang, W. Synthesis of efficient and reusable catalyst of size-controlle Au nanoparticles within a porous, chelating and intelligent hydrogel for aerobic alcohol oxidation. J. Mol. Catal. A Chem. 2010, 317, 81-88. [CrossRef]

37. Chen, X.; An, Y.; Zhao, D.; He, Z.; Zhang, Y.; Cheng, J.; Shi, L. Core-shell-corona au-micelle composites with a tunable smart hybrid shell. Langmuir 2008, 24, 8198-8204. [CrossRef] [PubMed]

38. Byun, J.; Huang, W.; Wang, D.; Li, R.; Zhang, K.A.I. $\mathrm{CO}_{2}$-Triggered Switchable Hydrophilicity of a Heterogeneous Conjugated Polymer Photocatalyst for Enhanced Catalytic Activity in Water. Angew. Chem. Int. Ed. 2018, 57, 2967-2971. [CrossRef] [PubMed]

39. Zhang, J.; Han, D.; Zhang, H.; Chaker, M.; Zhao, Y.; Ma, D. In situ recyclable gold nanoparticles using $\mathrm{CO}_{2}$-switchable polymers for catalytic reduction of 4-nitrophenol. Chem. Commun. 2012, 48, 11510-11512. [CrossRef] [PubMed]

40. Zhuang, Q.; Yang, Z.; Sobolev, Y.I.; Beker, W.; Kong, J.; Grzybowski, B.A. Control and Switching of Charge-Selective Catalysis on Nanoparticles by Counterions. ACS Catal. 2018, 8, 7469-7474. [CrossRef]

41. Elbert, J.; Mersini, J.; Vilbrandt, N.; Lederle, C.; Kraska, M.; Gallei, M.; Stühn, B.; Plenio, H.; Rehahn, M. Reversible activity modulation of surface-attached grubbs second generation type catalysts using redox-responsive polymers. Macromolecules 2013, 46, 4255-4267. [CrossRef]

42. Gallei, M.; Rüttiger, C. Recent Trends in Metallopolymer Design: Redox-Controlled Surfaces, Porous Membranes, and Switchable Optical Materials Using Ferrocene-Containing Polymers. Chem. A Eur. J. 2018, 24, 10006-10021. [CrossRef] [PubMed]

43. Neilson, B.M.; Bielawski, C.W. Illuminating photoswitchable catalysis. ACS Catal. 2013, 3, $1874-1885$. [CrossRef] 
44. Lawrence, R.L.; Scola, B.; Li, Y.; Lim, C.K.; Liu, Y.; Prasad, P.N.; Swihart, M.T.; Knecht, M.R. Remote Optically Controlled Modulation of Catalytic Properties of Nanoparticles through Reconfiguration of the Inorganic/Organic Interface. ACS Nano 2016, 10, 9470-9477. [CrossRef] [PubMed]

45. Szewczyk, M.; Sobczak, G.; Sashuk, V. Photoswitchable catalysis by a small swinging molecule confined on the surface of a colloidal particle. ACS Catal. 2018, 8, 2810-2814. [CrossRef]

46. Le Gong, L.; Yao, W.T.; Liu, Z.Q.; Zheng, A.M.; Li, J.Q.; Feng, X.F.; Ma, L.F.; Yan, C.S.; Luo, M.B.; Luo, F. Photoswitching storage of guest molecules in metal-organic framework for photoswitchable catalysis: Exceptional product, ultrahigh photocontrol, and photomodulated size selectivity. J. Mater. Chem. A 2017, 5, 7961-7967. [CrossRef]

47. Neri, S.; Martin, S.G.; Pezzato, C.; Prins, L.J. Photoswitchable catalysis by a nanozyme mediated by a lightsensitive cofactor. J. Am. Chem. Soc. 2017, 139, 1794-1997. [CrossRef] [PubMed]

48. Wei, Y.; Han, S.; Kim, J.; Soh, S.; Grzybowski, B.A. Photoswitchable catalysis mediated by dynamic aggregation of nanoparticles. J. Am. Chem. Soc. 2010, 132, 11018-11020. [CrossRef] [PubMed]

49. Zhao, H.; Sen, S.; Udayabhaskararao, T.; Sawczyk, M.; Kucanda, K.; Manna, D.; Kundu, P.K.; Lee, J.W.; Král, P.; Klajn, R. Reversible trapping and reaction acceleration within dynamically self-assembling nanoflasks. Nat. Nanotechnol. 2016, 11, 82-88. [CrossRef] [PubMed]

50. Mao, X.; Tian, W.; Wu, J.; Rutledge, G.C.; Hatton, T.A. Electrochemically responsive heterogeneous catalysis for controlling reaction kinetics. J. Am. Chem. Soc. 2015, 137, 1348-1355. [CrossRef] [PubMed]

51. Gao, D.; Zhou, H.; Cai, F.; Wang, D.; Hu, Y.; Jiang, B.; Cai, W.-B.; Chen, X.; Si, R.; Yang, F.; et al. Switchable $\mathrm{CO}_{2}$ electroreduction via engineering active phases of Pd nanoparticles. Nano Res. 2017, 10, 2181-2191. [CrossRef]

52. Burnat, D.; Kontic, R.; Holzer, L.; Steiger, P.; Ferri, D.; Heel, A. Smart material concept: Reversible microstructural self-regeneration for catalytic applications. J. Mater. Chem. A 2016, 4, 11939-11948. [CrossRef]

53. Nishihata, Y.; Mizuki, J.; Akao, T.; Tanaka, H.; Uenishi, M.; Kimura, M.; Okamoto, T.; Hamada, N. Self-regeneration of a Pd-perovskite catalyst for automotiv emission control. Nature 2002, 418, 164-167. [CrossRef] [PubMed]

54. Kwon, O.; Sengodan, S.; Kim, K.; Kim, G.; Jeong, H.Y.; Shin, J.; Ju, Y.W.; Han, J.W.; Kim, G. Exsolution trends and co-segregation aspects of self-grown catalyst nanoparticles in perovskites. Nat. Commun. 2017, 8, 1-7. [CrossRef] [PubMed]

55. Adijanto, L.; Balaji Padmanabhan, V.; Küngas, R.; Gorte, R.J.; Vohs, J.M. Transition metal-doped rare earth vanadates: A regenerable catalytic material for SOFC anodes. J. Mater. Chem. 2012, 22, 11396-11402. [CrossRef]

56. Tanaka, H. An intelligent catalyst: The self-regenerative palladium-perovskite catalyst for automotive emissions control. Catal. Surv. Asia 2005, 9, 63-74. [CrossRef]

57. Onn, T.M.; Monai, M.; Dai, S.; Fonda, E.; Montini, T.; Pan, X.; Graham, G.W.; Fornasiero, P.; Gorte, R.J. Smart Pd Catalyst with Improved Thermal Stability Supported on High-Surface-Area $\mathrm{LaFeO}_{3}$ Prepared by Atomic Layer Deposition. J. Am. Chem. Soc. 2018, 140, 4841-4848. [CrossRef] [PubMed]

58. Teschner, D.; Borsodi, J.; Wootsch, A.; Révay, Z.; Hävecker, M.; Knop-gericke, A.; Jackson, S.D.; Schlögl, R. The Roles of Subsurface Carbon. Science 2008, 320, 86-89. [CrossRef] [PubMed]

59. Armbrüster, M.; Behrens, M.; Cinquini, F.; Föttinger, K.; Grin, Y.; Haghofer, A.; Klötzer, B.; Knop-Gericke, A.; Lorenz, H.; Ota, A.; et al. How to Control the Selectivity of Palladium-based Catalysts in Hydrogenation Reactions: The Role of Subsurface Chemistry. ChemCatChem 2012, 4, 1048-1063. [CrossRef]

60. Niazov, T.; Shlyahovsky, B.; Willner, I. Photoswitchable electrocatalysis and catalyzed chemiluminescence using photoisomerizable monolayer-functionalized surfaces and Pt nanoparticles. J. Am. Chem. Soc. 2007, 129, 6374-6375. [CrossRef] [PubMed]

61. Mazur, T.; Lach, S.; Grzybowski, B.A. Heterogeneous Catalysis "on Demand": Mechanically Controlled Catalytic Activity of a Metal Surface. ACS Appl. Mater. Interfaces 2017, 9, 44264-44269. [CrossRef] [PubMed]

62. He, X.; Aizenberg, M.; Kuksenok, O.; Zarzar, L.D.; Shastri, A.; Balazs, A.C.; Aizenberg, J. Synthetic homeostatic materials with chemo-mechano-chemical self-regulation. Nature 2012, 487, 214-218. [CrossRef] [PubMed]

63. Vassalini, I.; Alessandri, I. "The phactalysts": Carbon nanotube/ $\mathrm{TiO}_{2}$ composites as phototropic actuators for wireless remote triggering of chemical reactions and catalysis. Nanoscale 2017, 9, 11446-11451. [CrossRef] [PubMed] 
64. Melchionna, M.; Beltram, A.; Stopin, A.; Montini, T.; Lodge, R.W.; Khlobystov, A.N.; Bonifazi, D.; Prato, M.; Fornasiero, P. Magnetic shepherding of nanocatalysts through hierarchically-assembled Fe-filled CNTs hybrids. Appl. Catal. B Environ. 2018, 227, 356-365. [CrossRef]

65. Li, Z.-X.; Luo, D.; Li, M.-M.; Xing, X.-F.; Ma, Z.-Z.; Xu, H. Recyclable Fe3O4 Nanoparticles Catalysts for Aza-Michael Addition of Acryl Amides by Magnetic Field. Catalysts 2017, 7, 219. [CrossRef]

66. Vinum, M.G.; Almind, M.R.; Engbæk, J.S.; Vendelbo, S.B.; Hansen, M.F.; Frandsen, C.; Bendix, J.; Mortensen, P.M. Dual-Function Cobalt-Nickel Nanoparticles Tailored for High-Temperature InductionHeated Steam Methane Reforming. Angew. Chem. Int. Ed. 2018, 57, 10569-10573. [CrossRef] [PubMed]

67. You, H.; Jia, Y.; Wu, Z.; Xu, X.; Qian, W.; Xia, Y.; Ismail, M. Strong piezo-electrochemical effect of multiferroic $\mathrm{BiFeO}_{3}$ square micro-sheets for mechanocatalysis. Electrochem. Commun. 2017, 79, 55-58. [CrossRef]

2018 by the authors. Licensee MDPI, Basel, Switzerland. This article is an open access article distributed under the terms and conditions of the Creative Commons Attribution (CC BY) license (http:/ / creativecommons.org/licenses/by/4.0/). 